Atiyah, M.F., und F. Hirzebruch

Math. Zeitschr. 77, 149-187 (1961)

\title{
Cohomologie-Operationen und charakteristische Klassen
}

Herrn FrIEdrich KarL Schmidr zum 60. Geburtstag am 22.9.1961 gewidmet

Von.

M. F. ATIYAH und F. HIRZEBRUCH

EINLEITUng. Bereits im Jahre 1950 hat WUWEN-Tsun [25] eine Beziehung zwischen den Steenrodschen Cohomologie-Operationen

$$
S q^{i}: H^{n}\left(X ; \boldsymbol{Z}_{2}\right) \rightarrow H^{n+i}\left(X ; \boldsymbol{Z}_{2}\right)
$$

und den Stiefel-Whitneyschen Klassen $w_{i} \in H^{i}\left(M ; \boldsymbol{Z}_{2}\right)$ einer kompakten differenzierbaren Mannigfaltigkeit $M$ hergestellt. Wu definiert Polynome $U_{j}\left(w_{1}, \ldots, w_{j}\right)$ vom Gewichte $j$ derart, daß in jeder kompakten differenzierbaren $m$-dimensionalen Mannigfaltigkeit $M$ gilt:

$$
S q^{i} v=U_{i} \cdot v \quad \text { für alle } v \in H^{m-i}\left(M ; \boldsymbol{Z}_{2}\right) .
$$

Wu hat seine Resultate auf die Steenrodschen Operationen $\mathscr{P}^{r}$ in der Cohomologie mit Koeffizienten $\boldsymbol{Z}_{q}$ ( $q$ ungerade Primzahl) und Pontrjaginsche Klassen (reduziert $\bmod q$ ) übertragen [26]. Im Jahre 1953 stellte einer der Verfasser einen merkwürdigen Zusammenhang her zwischen den Wuschen Polynomen und den aus der algebraischen Geometrie bekannten Toddschen Polynomen [10]. Die Note [10] enthält keine Beweise. Diese werden in der vorliegenden Arbeit nachgeholt. Die Beziehungen zwischen Wuschen und Toddschen Polynomen (Satz von Riemann-Roch), die in [10] einen recht formalen Charakter hatten, werden vertieft. Die Formel (1) wird auf Abbildungen $f: Y \rightarrow X$ von Mannigfaltigkeiten verallgemeinert im gleichen Sinne, wie GRoThENDIECK [6] den Satz von Riemann-Roch [11] verallgemeinert hat. Der Satz von Grothendieck hat ein differenzierbares Analogon [3]. ADAMS hat in [2] gezeigt, wie der Chernsche Charakter einęs Vektorraumbündels mit Cohomologie-Operationen zusammenhängt. Dies erlaubt uns, die vorhin erwähnte Beziehung zwischen Wuschen und Toddschen Polynomen besser zu verstehen. Durch die Arbeiten $[3,6]$ ist es ja klar geworden, daß die totale Toddsche Klasse $\sum_{j=0}^{\infty} T_{j}\left(c_{1}, \ldots, c_{j}\right)$ aufgefaßt als Element desrationalen Cohomologieringes des klassifizierenden Raumes der unitären Gruppe nach Anwendung des Thomschen Isomorphismus in den Chernschen Charakter eines "Bündels" über dem universellen Thomschen Komplex übergeht. Wir werden sehen, daß durch Reduktion modulo einer Primzahl der differenzierbare Satz von Riemann-Roch in die für Abbildungen verallgemeinerten Wuschen Formeln übergeht. 
Durch die Cobordisme-Arbeit von Tном [22] sind prinzipiell alle Relationen mod 2 zwischen den Stiefel-Whitneyschen Zahlen einer kompakten differenzierbaren Mannigfaltigkeit bekannt. In [8] hat DoLD gezeigt, daß die Wuschen Relationen zwischen den Stiefel-Whitneyschen Zahlen vollständig sind. Die Wuschen Relationen erhält man, wenn man in (1) für v Polynome in den $w_{;}$ einsetzt und $S q^{i} v$ auf Grund universeller Formeln, die ebenfalls von $\mathrm{Wu}$ stammen, berechnet. Nun hat MILNOR in [17] entscheidende Resultate über den Cobordisme-Ring orientierter Mannigfaltigkeiten bewiesen, die es prinzipiell ermöglichen, alle Relationen zwischen den Pontrjaginschen Zahlen, welche ja ganze Zahlen sind, aufzustellen. Es gibt da aber nicht nur Relationen modulo einer Primzahl, sondern auch modulo Potenzen einer Primzahl. Zum Beispiel zeigt der Indexsatz [11], daß für eine $M^{8}$ gilt: $7 p_{2}-p_{1}^{2} \equiv 0$ (45). Die Pontrjaginschen Zahlen erfüllen keine Relationen modulo 2. Wir zeigen, daß die analog zum Fall der Stiefel-Whitneyschen Klassen mit Hilfe der Steenrodschen Operationen $\mathscr{P}^{r}$ gebildeten Wuschen Relationen für eine ungerade Primzahl $q$ wieder vollständig sind, d.h. alle Relationen $\bmod q$ zwischen Pontrjaginschen Zahlen (natürlich nicht die Relationen modulo Potenzen von $q$ ) implizieren. Von MiLnor [17] stammt auch ein komplexes Analogon des Cobordisme-Ringes, das es ermöglicht, alle Relationen zwischen den Chernschen Zahlen aufzustellen. (Es ist dabei gleichgültig, ob man nur projektiv-algebraische Mannigfaltigkeiten oder allgemeiner schwach-fastkomplexe Mannigfaltigkeiten betrachtet, vgl. auch den Bericht [12].) Die Relationen modulo einer Primzahl (nicht die modulo der Potenzen einer Primzahl) können wieder mit Hilfe der Wuschen Methode erhalten werden. Aus dem Satz von RiemannRoch stammende Ganzzahligkeitssätze [3, 5] liefern Relationen für Chernsche Zahlen. Wir vermuten, $\mathrm{da} B$ dies alle Relationen sind. Die Vermutung wird dadurch bestärkt, daß diese Riemann-Roch-Relationen modulo einer Primzahl mit den Wuschen Relationen übereinstimmen, also vollständig sind. Zur Aufstellung aller. Relationen modula einer Primzahl benötigt man nur den elementareren Teil der Milnorschen Arbeit [17], nämlich die Existenz gewisser Mannigfaltigkeiten (5.5 i), ii) der vorliegenden Arbeit) und Aussagen über das Operieren der Steenrodschen Algebra auf der Cohomologie des universellen unitären Thomschen Komplexes $M \boldsymbol{U}$ (s. 5.4). Die Vollständigkeit der Wuschen Relationen ist auf Grund dieser Tatsachen sehr einfach einzusehen.

Wir fassen kurz den Inhalt der einzelnen Paragraphen zusammen: In §1 führen wir den Begriff des Cohomologie-Automorphismus ein. Die Milnorsche Arbeit [16] über die Struktur der Steenrodschen Algebra ermöglicht es uns, die Cohomologie-Automorphismen durch gewisse Potenzreihen zu charakterisieren (Satz 1.8). Der nächste Paragraph bringt Beziehungen zwischen Cohomologie-Automorphismen und charakteristischen Klassen von Vektorraumbündeln (s. z. B. Satz 2.7). In $\$ 3$ beweisen wir für Abbildungen $f: Y \rightarrow X$ von Mannigfaltigkeiten und einen beliebigen Cohomologie-Automorphismus den Satz 3.2, der für die konstante Abbildung $Y \rightarrow$ Punkt eine Verallgemeinerung der Wuschen Formel (1) ist. Es genügt 3.2 für Einbettungen und Projektionen zu beweisen. Der Beweis verläuft analog zu dem des differenzier- 
baren Riemann-Rochschen Satzes $[3,13]$, nur ist er viel einfacher. Die Diagonalmethode, mit der die Wuschen Formeln häufig hergeleitet werden $[1,10$, 15, 25, 26], wird nicht herangezogen. Stattdessen wird benutzt, daB eine differenzierbare Mannigfaltigkeit in eine Sphäre geeigneter Dimension einbettbar ist. In $\$ 4$ besprechen wir den Satz von ADAMs über den Chernschen Charakter und in $\$ 5$ die Vollständigkeit der Wuschen Relationen zwischen charakteristischen Zahlen.

\section{§1. Cohomologie-Automorphismen}

1.1. In diesem Paragraphen wird die singuläre Cohomologie-Theorie mit Koeffizienten in dem Primkörper $\boldsymbol{Z}_{q}$ der Charakteristik $q(q \neq 0)$ betrachtet. Im allgemeinen wird die Primzahl $q$ festgewählt, so daß wir den Koeffizientenbereich $\boldsymbol{Z}_{q}$ bei der Anschreibung der Cohomologie-Gruppen weglassen werden, wenn keine Verwechslungen zu befürchten sind. Für einen topologischen Raum $X$ bezeichnet $H^{* *}(X)$ das direkte Produkt der $H^{i}(X)$. Ein Element $z \in H^{* *}(X)$ kann auf eine und nur eine Weise als "unendliche Summe"

$$
z=\sum_{i=0}^{\infty} z_{i}, \quad z_{i} \in H^{i}(X)
$$

geschrieben werden. Für eine stetige Abbildung $f: Y \rightarrow X$ bezeichnet $f^{* *}$ den induzierten Homomorphismus $H^{* *}(X) \rightarrow H^{* *}(Y)$. Für einen Raum $X$, dessen Cohomologie-Gruppen oberhalb einer gewissen Dimension $n_{0}$ alle verschwinden, können $H^{*}(X)$ und $H^{* *}(X)$ identifiziert werden. (Vgl. [5, Teil I, §6.1]; diese Bezeichnungen verwenden wir natürlich auch für andere Koeffizientenbereiche als $\boldsymbol{Z}_{q}$.)

Definition. Ein Cohomologie-Automorphismus $\lambda$ (für die Cohomologie mit Koeffizienten in $\boldsymbol{Z}_{q}$ ) ist eine Operation, die jedem topologischen Raum $X$ einen RingHomomorphismus

so zuordnet, daß

$$
\lambda: H^{* *}(X) \rightarrow H^{* *}(X)
$$

(1) $f^{* *} \lambda=\lambda f^{* *}$ für jede stetige Abbildung $f: Y \rightarrow X$ topologischer Räume $Y, X$, (2) $\lambda=$ Identität für $X=\boldsymbol{S}^{\mathbf{\lambda}}$ (eindimensionale Sphäre).

Es wird natürlich nicht verlangt, daß $\lambda$ die Graduierung erhält. Für eine ungerade Primzahl $q$ ist

$$
\mathscr{P}=\sum_{r=0}^{\infty} \mathscr{P}^{r}
$$

ein Cohomologie-Automorphismus, wo

$$
\mathscr{P}^{r}: H^{n}(X) \rightarrow H^{n+2 r(q-1)}(X)
$$

die $r$-te Steenrodsche reduzierte Potenz für die Primzahi $q$ ist [20]. Für $q=2$ ist entsprechend

$$
S q=\sum_{i=0}^{\infty} S q^{i}
$$


ein Cohomologie-Automorphismus, wo

$$
S q^{i}: H^{n}(X) \rightarrow H^{n+i}(X)
$$

das $i$-te Steenrodsche reduzierte Quadrat ist (vgl. auch [21]). Durch (2) wird z. B. die Operation $\lambda(x)=x^{q}\left(x \in H^{* *}\left(X ; \boldsymbol{Z}_{q}\right)\right)$ ausgeschlossen.

Es wird später leicht folgen, daß $\lambda: H^{* *}(X) \rightarrow H^{* *}(X)$ ein Isomorphismus ist. (Deshalb sprechen wir bereits jetzt von Cohomologie-Automorphismen.) Wir hätten auch allgemeinere Koeffizientenbereiche als die Primkörper der Charakteristik $q$ für die Untersuchung von Cohomologie-Automorphismen zugrunde legen können. Manche der folgenden Sätze blieben dann erhalten. Aber da das für die Zwecke der vorliegenden Arbeit nicht erförderlich ist, haben wir uns der Bequemlichkeit wegen auf die Primkörper beschränkt. Den Primkörper $\boldsymbol{Q}=\boldsymbol{Z}_{0}$ der Charakteristik 0 haben wir ausgeschlossen, da für ihn jeder Cohomologie-Automorphismus die Identität ist.

1.2. Ein Cohomologie-Automorphismus $\lambda$ ist bereits definiert, wenn man ihn für alle $C W$-Komplexe so definiert hat, daß alle Bedingungen der Definition 1.1 im 'Rahmen der Kategorie der CW-Komplexe [24] erfüllt sind. An Stëlle der $C W$-Komplexe könnte man auch die simplizialen Komplexe wählen. Eine entsprechende Bemerkung über Cohomologie-Operationen findet sich bereits bei SeRRE $[19, \S 4]$, der dort den allgemeinen Begriff der Cohomologie-Operation zuerst eingeführt hat. Wir wollen diese Dinge hier nicht näher ausführen. Wir erinnern nur daran, daß die geometrische Realisierung $|K|$ eines semisimplizialen Komplexes $K$ ein $C W$-Komplex ist [14]. Wenn $K$ insbesondere der singuläre Komplex $S X$ eines topologischen Raumes ist, dann hat man eine kanonische Abbildung $j_{X}:|S X| \rightarrow X$, welche Isomorphismen aller CohomologieGruppen induziert. Für eine stetige Abbildung $f: Y \rightarrow X$ hat man eine stetige Abbildung $f^{\prime}:|S Y| \rightarrow|S X|$ und das kommutative Diagramm:

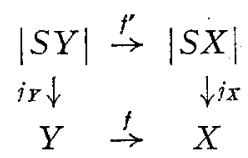

Wegen der Abbildung $j_{X}$ weiß man also, wie $\lambda$ auf $H^{* *}(X)$ zu definieren ist, wenn man es auf $H^{* *}(|S X|)$ kennt. Man verwendet (3), um zu zeigen, daß der nun für alle topologischen Räume definierte Cohomologie-Automorphismus $\lambda$ mit Abbildungen vertauschbar ist.

Wir stellen weiter fest, daß ein Cohomologie-Automorphismus $\lambda$ sogar bereits definiert ist, wenn er fïr alle endlichen $C W$-Komplexe so definiert ist, daß alle Bedingungen der Definition $1.1 \mathrm{im}$ Rahmen der Kategorie der endlichen $\mathrm{CW}$ Komplexe erfïllt sind.

Für jeden $C W$-Komplex $X$ hat man nämlich die Isomorphie

$$
H^{* *}\left(X ; \boldsymbol{Z}_{q}\right)=\lim _{\leftarrow} H^{*}\left(X_{\alpha} ; \boldsymbol{Z}_{q}\right),
$$


wo $X_{\alpha}$ alle endlichen Teilkomplexe durchläuft (inverser Limes). Für die Isomorphie (4) wird ausgenutzt, daß der Koeffizientenbereich $\boldsymbol{Z}_{q}$ ein $\mathrm{Körper}$ ist. (Vgl. hierzu [9, Chap. VIII, Theorem 5.7 und Exercise F 2].)

Die Überlegungen dieses Abschnitts zeigen, daß gewisse im nächsten $A b$ schnitt zu formulierende Eigenschaften von $\lambda$ nur für endliche $C W$-Komplexe bewiesen zu werden brauchen.

1.3. Zunächst eine Bemerkung über das Cup-Produkt. Für eine Triade $\left(X ; Y_{1}, Y_{2}\right)$ topologischer Räume $\left(Y_{1}<X, Y_{2}<X\right)$ kann man die singulären (semi-simplizialen) Komplexe $S Y_{1} \cup S Y_{a}$ und $S\left(Y_{1} \cup Y_{2}\right)$ betrachten, welche Unterkomplexe von $S X$ sind. Die Triade $\left(X ; Y_{1}, Y_{2}\right)$ heiBe eigentlich, wenn die Inklusion $S Y_{1} \cup S Y_{2}<S\left(Y_{1} \cup Y_{2}\right)$ Isomorphismen der ganzzahligen Homologiegruppen dieser Komplexe und damit auch Isomorphismen aller Homologieund Cohomologiegruppen mit beliebigen Koeffizienten induziert [ 9 , Chap. VIJ, \$11]. Zum Beispiel ist $\left(X ; Y_{1}, Y_{2}\right)$ dann eigentlich, wenn $X$ ein $C W$-Komplex ist und $Y_{1}, Y_{2}$ Unterkomplexe sind. Verwendet man einen Ring als Koeffizientenbereich für die singuläre Cohomologie, dann ist für eine eigentliche Triade $\left(X ; Y_{1}, Y_{2}\right)$ das Cup-Produkt definiert: Für $a \in H^{n}\left(X, Y_{1}\right)$ und $b \in H^{m}\left(X, Y_{2}\right)$ ist $a \cup b=a b$ ein Element von $H^{n+m}\left(X, Y_{1} \cup Y_{2}\right)$. Die übliche Definition des CupProdukts liefert $t^{k} a b$ zunächst nur als Element von $H^{n+m}\left(S X, S Y_{1} \cup S Y_{2}\right)$. Diese Gruppe ist aber bei einer eigentlichen Triade kanonisch isomorph $\mathrm{zu}$ $H^{n+m}\left(S X, S\left(Y_{1} \cup Y_{2}\right)\right)=H^{n+m}\left(X, Y_{1} \cup Y_{2}\right)$. (Man ziehe das Fünfer-Lemma heran.)

SATZ. Ein Cohomologie-Automorphismus $\lambda$ fïr die Cohomologie mit Koeffizienten $\boldsymbol{Z}_{q}$ sei gegeben (1.1). Er kann dann auf eine und nur eine Weise für beliebige Raumpaare $(X, Y)$ definiert werden,

$$
\lambda: H^{* *}(X, Y) \rightarrow H^{* *}(X, Y)
$$

so daß er für $Y=\varnothing$ mit dem gegebenen $\lambda$ übereinstimmt und daß gilt:

(i) $\lambda$ ist additiv.

(ii) $\lambda$ ist mit Abjildungen $(X, Y) \rightarrow\left(X^{\prime}, Y^{\prime}\right)$ vertauschbar.

(iii). Für eine eigentiche Triade $\left(X ; Y_{1}, Y_{2}\right)$, für $a \in H^{* *}\left(X, Y_{1}\right), b \in H^{* *}\left(X, Y_{2}\right)$ und damit $a \cup b \in H^{* *}\left(X, Y_{1} \cup Y_{2}\right)$ gilt: $\lambda(a \cup b)=\lambda a \cup \lambda b$.

(iv) $\lambda$ ist stabil.

(v) $\lambda$ ist mit dem Corand-Operator $\delta: H^{* *}(Y) \rightarrow H^{* *}(X, Y)$ vertatischbar.

BeweIs. Für ein Paar $(X, Y)$ endlicher $C W$-Komplexe ( $Y$ Teilkomplex) ist

$$
H^{*}(X, Y)=\tilde{H} *(X / Y)
$$

wo $X / Y$ aus $X$ durch Identifikation von $Y$ zu einem Punkt ensteht, der in $X / Y$ die Rolle des Basispunktes übernimmt. $\widetilde{H}^{*}$ bezeichnet die bezüglich des Basispunktes ,reduzierte" Cohomologie $\left(\widetilde{H}^{i}=H^{i}\right.$ für $\left.i>0\right)$. Damit ist klar, wie $\lambda$ für ein Paar endlicher $C W$-Komplexe definiert werden muß. Für ein beliebiges Paar $(X, Y)$ von $C W$-Komplexen ( $Y$ Teilkomplex) gilt in Verallgemeinerung 
von (4)

$$
H^{* *}(X, Y)=\lim _{\leftarrow} H^{*}\left(X_{\alpha}, Y \cap X_{\alpha}\right),
$$

wo $X_{\alpha}$ die endlichen Teilkomplexe durchläuft. $\lambda$ wird nach (6) für $(X, Y)$ definiert. Man sieht leicht, daß die Definition nicht davon abhängt, wie der Raum $X$ als $C W$-Komplex dargestellt wird und daß $\lambda$ mit Abbildungen innerhalb der Kategorie der Paare von $C W$-Komplexen vertauschbar ist. (Vgl. die entsprechenden Überlegungen bei M. F. ATIYAH and F. HIRZEBRUCH, Analytic cycles on complex manifolds (erscheint demnächst in ",Topology“, Pergamon Press), §1). Für ein beliebiges Paar $(X, Y)^{\prime}$ topologischer Räume ist $S Y$ Teilkomplex von $S X$ und

$$
i_{X}:(|S X|,|S Y|) \rightarrow(X, Y)
$$

induziert Isomorphismen der Cohomologie-Gruppen. Damit ist $\lambda$ für ein beliebiges Paar definiert. (ii) folgt unmittelbar aus (3). Nun zum Beweis von (iii). Für Paare $\left(X_{1}, Y_{1}\right)$ und $\left(X_{2}, Y_{2}\right)$ endlicher $C W$-Komplexe betrachten wir die Homomorphismen

$$
\left\{\begin{array}{c}
H^{*}\left(X_{1}, Y_{1}\right) \otimes H^{*}\left(X_{2}, Y_{2}\right) \stackrel{\propto}{\rightarrow} H^{*}\left(X_{1} \times \underset{X_{2}}{X_{2}}, Y_{1} \times X_{2} \cup X_{1} \times Y_{2}\right) \\
\psi^{\beta} \\
H^{*}\left(\left(X_{1} / Y_{1}\right) \times\left(X_{2} / Y_{2}\right)\right)
\end{array}\right.
$$

(Tensorprodukt über $\boldsymbol{Z}_{q}$ ), wobei $\alpha$ und $\beta \circ \alpha$ in bekannter Weise durch das Cup-Produkt gegeben werden, während $\beta$ durch

$$
X_{1} / Y_{1} \times X_{2} / Y_{2} \rightarrow\left(X_{1} / Y_{1} \times X_{2} / Y_{2} /\right)\left(X_{1} / Y_{1} \vee X_{2} / Y_{2}\right)=X_{1} \times X_{2} /\left(Y_{1} \times X_{2} \cup X_{1} \times Y_{2}\right)
$$

induziert wird (vgl. $\lfloor 4, \S 1]$ ). Bezeichnet man mit $p_{1}$ und $p_{2}$ die Projektionen von $X_{1} / Y_{1} \times X_{2} / Y_{2}$ auf $X_{1} / Y_{1}$ bzw. $X_{2} / Y_{2}$, dann ist $(\beta \circ \&)\left(x_{1} \otimes x_{2}\right)=p_{1}^{*} x_{1} \cup p_{2}^{*} x_{2}$. Daraus folgt, daß $\lambda$ mit $\beta \circ \alpha$ vertauschbar ist, wobei wir $\lambda$ auf dem in (8) vorkommenden Tensorprodukt durch $\lambda\left(x_{1} \otimes x_{2}\right)=\lambda x_{1} \otimes \lambda x_{2}$ operieren lassen. Da $\beta$ injektiv und mit $\lambda$ vertauschbar ist, ist $\lambda$ auch mit $\alpha$ vertauschbar. Wir setzen nun $X_{1}=X_{2}=X$. Die Diagonalbildung von $X$ in $X \times X$ bildet $Y_{1} \cup Y_{2}$ in $Y_{1} \times X_{2} \cup X_{1} \times Y_{2}$ ab.

$$
\Delta:\left(X, Y_{1} \cup Y_{2}\right) \rightarrow\left(X \times X, Y_{1} \times X \cup X \times Y_{2}\right) .
$$

Die zu beweisende Gleichung (iii) ist nichts anderes als die Vertauschbarkeit von $A^{*} \circ \alpha$ mit $\lambda$, die aber richtig ist, da $\lambda$ mit $\alpha$ und mit $\Delta^{*}$ vertauschbar ist. Man beweist (iii) in voller Allgemeinheit, indem man (6) und die Realisierungen semi-simplizialer Komplexe verwendet. Die Einzelheiten mögen dem Leser überlassen sein.

Wir kommen nun zum Beweis von (iv). Es sei $X$ ein endlicher $C W$-Komplex mit Basispunkt. Die 1-Sphäre $\boldsymbol{S}^{\mathbf{1}}$ werde kanonisch orientiert und sei ebenfalls mit Basispunkt versehen. Unter dem Koeffizienten-Homomorphismus $\boldsymbol{Z} \rightarrow \boldsymbol{Z}_{q}$ geht das zur Orientierung gehörige erzeugende Element von $H^{\mathbf{1}}\left(\boldsymbol{S}^{\mathbf{1}} ; \boldsymbol{Z}\right)$ in ein erzeugendes Element $g$ von $H^{1}\left(\boldsymbol{S}^{1} ; \boldsymbol{Z}_{q}\right)$ über. Die Einhängung von $X$ ist der 
$C W$-Komplex

$$
\mathbf{S}(X)=\mathbf{S}^{1} \wedge X=\boldsymbol{S}^{1} \times X / \mathbf{S}^{1} \vee X, \quad \text { vgl. }[4, \S 1] .
$$

Man hat einen kanonischen Isomorphismus

$$
E: \widetilde{H}^{*}(X) \rightarrow \widetilde{H}^{*}\left(\mathbf{S}^{1} \wedge X\right),
$$

der wie folgt definiert ist. Man setzt in (8) $X_{1}=\mathbf{S}^{1}, X_{2}=X$ und für $Y_{1}, Y_{2}$ den jeweiligen Basispunkt. Dann ist

$$
E(x)=\alpha(g \otimes x), \quad x \in \widetilde{H}^{*}(X)=H^{*}\left(X_{2}, Y_{2}\right) .
$$

Wie wir vorhin bemerkt haben, ist $\lambda$ mit $\alpha$ vertauschbar. Nach (2) ist $\lambda(g)=g$. Also ist $\lambda$ mit $E$ vertauschbar. Dies ist gerade die Stabilität.

(v) braucht wegen (6) und (7) nur für ein Paar $(X, Y)$ endlicher $C W$-Komplexe bewiesen zu werden. In $Y$ werden ein Basispunkt ausgezeichnet. Es genügt dann, die Behauptung für $y \in \widetilde{H}^{*}(Y)$ nachzuweisen. Es sei $f$ die Einbettung von $Y$ in $X$. Der Abbildungskegel $C_{f}$ ist ein endlicher $C W$-Komplex mit Basispunkt und auf kanonische Weise homotopie-äquivalent mit $X / Y$, vg1. $[4,18]$. Es gibt eine Abbildung $Q f$ von $C_{f}$ in $\mathbb{S}^{1} \wedge Y$ und $\delta$ ist gleich $(Q f)^{*} \circ E$ gefolgt von dem kanonischen Isomorphismus $\widetilde{H}^{*}\left(C_{f}\right) \cong \tilde{H}^{*}(X / Y)=H^{*}(X, Y)$. Die Behauptung (v) folgt nun aus (1) und der vorhin bewiesenen Vertauschbarkeit von $\lambda$ mit $E$.

1.4. Ein Cohomologie-Automorphismus $\lambda$ ist bereits durch sein Operieren auf den Cohomologie-Ringen der endlichen $C W$-Komplexe bestimmt (1.2). Aus dieser Tatsache und aus 1.3 (iv) folgert man, daß sich $\lambda$ in der Form

$$
\lambda=\sum_{j=0}^{\infty} \lambda_{j}
$$

schreiben läßt, wo $\lambda_{j}: H^{n}(X) \rightarrow H^{n+j}(X)$, (X beliebiger topologischer Raum, $n=0,1,2, \ldots)$, eine stabile Operation ist, die die Dimension um $j$ erhöht. (9) bedeutet, daß für $z \in H^{* *}(X)$ mit $z=\sum_{i=0}^{\infty} z_{i}$ und $z_{i} \in H^{i}(X)$

und

$$
\lambda_{j}(z)=\sum_{i=0}^{\infty} \lambda_{j} z_{i}
$$

$$
\lambda(z)=\sum_{j=0}^{\infty} \lambda_{j} z .
$$

Auf Grund der Definition von $H^{* *}$ sind die unendlichen Summen (10) und (11) sinnvoll.

Auf $H^{*}\left(\mathbf{S}^{n}\right)$ ist $\lambda$ gleich der Identität ((2) und 1.3 (iv): Vertauschbarkeit mit $E$, die $n$-Sphäre $\boldsymbol{S}^{n}$ ist die $(n-1)$-fache Einhängung von $\left.\boldsymbol{S}^{\mathbf{1}}\right)$. Für einen endlichen $C W$-Komplex $X$, dessen $n$-Skelett mit $X^{n}$ bezeichnet werde, ist $\lambda$ auf $H^{*}\left(X^{n}, X^{n-1}\right)$ die Identität, da $X^{n} / X^{n-1}$ ein Bukett von Sphären ist. 
Wegen der exakten Sequenzen

$$
H^{n}\left(X^{n}, X^{n-1}\right) \rightarrow H^{n}\left(X^{n}\right) \rightarrow 0, \quad 0 \rightarrow H^{n}(X) \rightarrow H^{n}\left(X^{n}\right)
$$

ist $\lambda_{0}$ die Identität (und zwar wegen 1.2 für jeden topologischen $\operatorname{Raum} X$ ).

Lemma. Für jeden Raum $X$ ist $\lambda$ ein Automorphismus von $H^{* *}(X)$.

BEwEIs. Wegen 1.2 genügt es, dies für endliche $C W$-Komplexe nachzuweisen, da dann die Existenz des inversen Cohomologie-Automorphismus $\lambda^{-1}$ gesichert ist. In diesem Fall ist die Behauptung klar, da $\lambda$ wegen (9) und $\lambda_{0}=$ Identität injektiv ist und $H^{* *}(X)=H^{*}(X)$ ein endlich-dimensionaler Vektorraum über $\boldsymbol{Z}_{q}$ ist. Man kann auch $\lambda^{-1}$. sofort explizit angeben. Man setze

Dann ist

$$
\lambda=1+\sum_{i=1}^{\infty} \lambda_{i}=1+\mu
$$

$$
\lambda^{-1}=\sum_{j=0}^{\infty}(-1)^{i} \mu^{j},
$$

wo $\mu^{j}$ die $j$-fache Iteration von $\mu$ bedeutet. Es ist klar, daß (12) die $j$-dimensionale Komponente von $\lambda^{-1}$ als endliche Summe

iefert.

$$
\sum a_{i_{1} \ldots i_{r}} \lambda_{i_{1}} \circ \cdots \circ \lambda_{i_{r}} \quad\left(i_{1}+\cdots+i_{r}=j, a_{i_{1}, \ldots i_{r}} \in \mathbf{Z}_{q}\right)
$$

Cohomologie-Automorphismen können hintereinander geschaltet werden. Die Cohomologie-Automorphismen (für. die Cohomologie mit Koeffizienten in $\boldsymbol{Z}_{q}$ ) bilden eine Gruppe, die mit $\operatorname{Coh}\left(\boldsymbol{Z}_{q}\right)$ bezeichnet werde.

1.5. SaTz. Wenn $q \neq 2$, dann sind für einen Cohomologie-Automorphismus $\lambda$ alle Komponenten $\lambda_{i}$ (vgl. (9)) mit ungeradem $i$ gleich 0.

Beweis. $H^{* *}\left(X \times \mathbf{S}^{1}\right)$ enthält $H^{* *}(X)$ als Unterring und ist ein freier Modul über $H^{* *}(X)$ mit einer Basis 1, $g \in H^{* *}\left(X \times \boldsymbol{S}^{1}\right) ;\left(g \in H^{1}, g g=0\right)$, für die $\lambda(g)=g$. Für $x \in H^{m}(X)$ ist

$$
\lambda(x g)=\lambda x \cdot g=(-1)^{m} \lambda(g x)=(-1)^{m} g \cdot \lambda x=(-1)^{m} \sum_{i=0}^{\infty}(-1)^{i+m} \lambda_{i} x \cdot g .
$$

Es folgt $\lambda_{i}=(-1)^{i} \lambda_{i}$ und damit die Behauptung.

Der Satz ist für $q=2$ falsch, wie der Cohomologie-Automorphismus $S q$ (s. 1.1) zeigt.

1.6. Zusammenfassend können wir sagen, daß ein Cohomologie-Automor phismus $\lambda$ (für die Cohomologie mit Koeffizienten in $\boldsymbol{Z}_{q}$ ) eine natürliche Transformation der singulären Theorie in sich ist, welche die multiplikative Struktur und für $q \neq 2$ die $\boldsymbol{Z}_{2}$-Graduierung erhält:

$$
\left\{\begin{array}{l}
H^{* *}(X)=H^{e v}(X)+H^{o d}(X), \\
H^{e v}(X)=\prod_{m=0}^{\infty} H^{2 m}(X), \quad H^{o d}(X)=\prod_{m=0}^{\infty} H^{2 m+1}(X), \quad \text { (vgl. [4, §1.10]) }
\end{array}\right.
$$

Wir unterscheiden jetzt die beiden Fälle $q$ ungerade und $q=2$. 
a) qungerade.

Der Ring der formalen Potenzreihen in einer Unbestimmten $x$ über $\mathscr{Z}_{q}$ werde mit $\boldsymbol{Z}_{q}[[x]]$ bezeichnet (entsprechende Bezeichnung für mehrere Unbestimmte). $\boldsymbol{Z}_{q}[[x]]$ ist isomorph zu $H^{* *}\left(\boldsymbol{P}_{\infty}(\boldsymbol{C})\right)$, dem Cohomologie-Ring des unendlich-dimensionalen komplexen projektiven Raumes. Wir stiften einen Isomorphismus, indem wir $x$ ein von 0 verschiedenes (und damit erzeugendes) Element von $H^{2}\left(\boldsymbol{P}_{\infty}(\boldsymbol{C})\right) \cong \boldsymbol{Z}_{q}$ zuordnen. Jetzt ist $\lambda(x)$ wohldefiniert als Element von $\boldsymbol{Z}_{q}[[x]]$, und $z$ war ist es wegen $\lambda_{0}=$ Identität von der Form

$$
\lambda(x)=\sum_{j=1}^{\infty} b_{j} x^{j}, \quad b_{j} \in Z_{q}, \quad b_{1}=1 .
$$

Da $\boldsymbol{P}_{\infty}(\boldsymbol{C})$ ein Eilenberg-MacLanescher Raum $K(\boldsymbol{Z}, 2)$ ist, gilt (14), wenn man $x$ durch ein beliebiges Element von $H^{2}(X, Y)$ ersetzt, welches Reduktion einer ganzzahligen 2-dimensionalen Cohomologie-Klasse von $(X, Y)$ ist. Insbesondere trifft das zu für $X=\boldsymbol{P}_{\infty}(\boldsymbol{C}) \times \boldsymbol{P}_{\infty}(\boldsymbol{C})$ und $Y=\varnothing$. Nun ist

$$
H^{* *}\left(\boldsymbol{P}_{\infty}(\boldsymbol{C}) \times \boldsymbol{P}_{\infty}(\boldsymbol{C})\right)=\boldsymbol{Z}_{q}\left[\left[x_{1}, x_{2}\right]\right] \text {, }
$$

wo $x_{1}, x_{2}$ Unbestimmte über $\boldsymbol{Z}_{q}$ sind, die bei dem Isomophismus 2-dimensionalen Cohomologieklassen entsprechen. Aus der Additivität von $\lambda$ ergibt sich folgende Gleichung in $\boldsymbol{Z}_{q}\left[\left[x_{1}, x_{2}\right]\right]$

$$
\sum_{j=1}^{\infty} b_{j}\left(x_{1}+x_{2}\right)^{j}=\sum_{j=1}^{\infty} b_{j} x_{1}^{j}+\sum_{j=1}^{\infty} b_{j} x_{2}^{j} .
$$

Elementare Eigenschaften der Binomialkoeffizienten implizieren, daß $b_{j}=0$, wenn $j$ keine Poten $z$ von $q$ ist. Wir setzen

$$
\lambda(x)=x+a_{1} x^{q}+a_{2} x^{q^{2}}+\cdots+a_{j} x^{q}+\cdots, a_{j} \in Z_{q} .
$$

Die Potenzreihe $\lambda(x) \in \boldsymbol{Z}_{q}[[x]]$ hängt offenbar nicht von der Auswahl des erzeugenden Elementes von $H^{2}\left(P_{\infty}(\boldsymbol{C})\right)$ ab, das wir $x$ zugeordnet haben.

b) $q=2$.

Der Ring $\boldsymbol{Z}_{2}[[x]]$ wird jetzt als Cohomologie-Ring $H^{* *}\left(\boldsymbol{P}_{\infty}(\boldsymbol{R})\right)$ des unendlich-dimensionalen reellen projektiven Raumes realisiert, indem wir der Unbestimmten $x$ das von 0 verschiedene Element von $H^{\mathbf{1}}\left(\boldsymbol{P}_{\infty}(\boldsymbol{R})\right) \cong \boldsymbol{Z}_{2}$ zuordnen. Wie in a) erhält man die Potenzreihe

$$
\lambda(x)=x+a_{1} x^{2}+a_{2} x^{4}+\cdots+a_{j} x^{2^{j}}+\cdots, a_{j} \in Z_{2} .
$$

Die Gleichung (17) bleibt richtig, wenn man $x$ durch ein beliebiges Element von $H^{\mathbf{1}}(X, Y)$ ersetzt, da $\boldsymbol{P}_{\infty}(\boldsymbol{R})$ ein Eilenberg-MacLanescher $\operatorname{Raum} K\left(\boldsymbol{Z}_{2}, 1\right)$ ist.

Bei der Definition der Potenzreihe $\lambda(x)$ wurde die Additivität von $\lambda$ benutzt, aber nicht, daß $\lambda$ die multiplikative Struktur erhälk.

Mit $G\left(\boldsymbol{Z}_{q}\right)$ werde die Gruppe der Potenzreihen der Gestalt (16) bzw. (17) bezüglich des Ineinander-Setzens bezeichnet:

$$
f(x) \circ g(x)=f(g(x))
$$


Ordnet man jedem Cohomologie-Automorphismus $\lambda$ die Potenzreihe $\dot{\lambda}(x)^{*} \mathrm{zu}$, dann erhält man einen Homomorphismus

$$
\operatorname{Coh}\left(\boldsymbol{Z}_{q}\right) \rightarrow G\left(\boldsymbol{Z}_{q}\right), \quad \text { (vgl. SchluB von 1.4). }
$$

Wir werden in 1.8 sehen, daß (18) bijektiv, also ein Isomorphismus ist. Entscheidend sind dafür die Ergebnisse von Milnor über die Steenrodsche Algebra.

1.7. Über die Steenrodsche Algebra. Wir folgen den Bezejchnungen von Milnor [16]. Es sei also $S^{*}$ die Steenrodsche Algebra für die Primzahl $q$. Die Algebra $S^{*}$ ist graduiert, assoziativ und vom endlichen Typ über $\boldsymbol{Z}_{q}$, d.h.

$$
S^{*}=\sum_{i=0}^{\infty} S^{i}
$$

und $S^{i}$ ist ein endlich-dimensionaler Vektorraum über $\boldsymbol{Z}_{q}$. ( $S^{0}$ hat die Dimension 1.) Nach Cartan und SerRe $[7,19]$ ist $S^{i}$ die additive Gruppe der stabilen Operationen vort der Cohomologie mit Koeffizienten $\boldsymbol{Z}_{q}$ in sich, die die Dimension um $i$ erhöhen. Also ist für jeden Cohomologie-Automorphismus $\lambda$ die Komponente $\lambda_{i}$ ein Element von $S^{i}$. Jedoch ist $\lambda$ selbst nicht ein Element von $S^{*}$. Wir führen deshalb in Analogie zu 1.1 das direkte Produkt der $S^{i}$ ein und nennen es $S^{* *}$. Die Cohomologie-Automorphismen entsprechen dann bestimmten Elementen von $S^{* *}$. Es handelt sich nun darum, festzustellen, welche Elemente von $S^{* *}$ Cohomologie-Automorphismen sind.

MILNoR definiert in $\S 3$ seiner Arbeit [16] den Ring-Homomorphismus

$$
\psi^{*}: S^{*} \rightarrow S^{*} \otimes S^{*} \quad \text { (Tensorprodukte immer über } \boldsymbol{Z}_{q} \text { ), }
$$

welcher $S^{*}$ zu einer Hopfschen Algebra macht. Wir setzen $S^{*} \otimes S^{*}=U^{*}$. Dann ist

$$
U^{n}=\sum_{i+j=n} S^{i} \otimes S^{j}
$$

und $\psi^{*}$ bildet $S^{n}$ in $U^{n}$ ab. Es ist klar, daß $\psi^{*}$ auf natürliche Weise einen Homomorphismus

$$
\psi^{* *}: S^{* *} \rightarrow U^{* *}, \quad U^{* *}=\prod_{n=0}^{\infty} U^{n},
$$

induziert. Wir nennen nun diejenigen Elemente $c$ von $S^{* *}$ multiplikativ, welche von 0 verschieden sind und für die

das soll heißen

$$
\psi^{* *} c=c \otimes c
$$

$$
\left.\psi^{*} c_{k}=\sum_{i+j=k} c_{i} \otimes c_{j}, \quad \text { (vgl. auch }[1]\right)
$$

Da $S^{*}$ eine Hoṕfsche Algebra ist, folgt sofort $c_{0}=1$.

Lemma. Für ungerades $q$ ist ein Element von $S^{* *}$ dann und nur dann ein Cohomologie-A utomorphismus, wenn es multiplikativ ist und wenn seine ungerade-dimensionalen Komponenten verschwinden. Für $q=2$ ist ein Element von 
$S^{* *}$ dann und nur dann ein Cohomologie-Automorphismus, wenn es maltiplikativ ist.

Der Beweis ergibt sich sofort aus 1.5 und aus der Definition von $\psi^{*}$ (s. [16, §3, Lemma 1]). Wir hätten den Begriff des Cohomologie-Automorphismus auch so fassen können, daß alle multiplikativen Elemente von $S^{* *}$, auch diejenigen, deren ungerade-dimensionalen Komponenten nicht alle verschwinden, darunterfallen. Aber der Einfachheit wegen haben wir uns für $q \neq 2$ auf die beschränkt, welche die $\boldsymbol{Z}_{2}$-Graduierung erhalten (1.6) oder (äquivalent) auf die, welche Ring-Homomorphismen $H^{* *}(X) \rightarrow H^{* *}(X)$ sind.

1.8. SATZ. Der Homomorphismus $\operatorname{Coh}\left(\boldsymbol{Z}_{q}\right) \rightarrow G\left(\boldsymbol{Z}_{q}\right)$ der Gruppe der Cohomologie-Automorphismen in die Gruppe $G\left(\boldsymbol{Z}_{q}\right)$ (s. $1.6(18)$ ) ist bijektiv.

BEWEIS FÜR UNGERADES $q$ : MILNOR [16] betrachtet das Dual $S_{*}$ der Steenrodschen Algebra $S^{*}$. Da $S^{* *}=\operatorname{Hom}\left(S_{*}, \boldsymbol{Z}_{q}\right)$, hat man die Paarung $\left\langle\boldsymbol{a}, a^{r}\right\rangle$ $z$ wischen Elementen $a \in S^{* *}$ und $a^{\prime} \in S_{*}$, die von Milnor nur für $a \in S^{*}$ betrachtet wird. Für homogen-dimensionale Elemente $a, b \in S^{*}$ und $a^{\prime}, b^{\prime} \in S_{*}$ gilt

$$
\left\langle a \otimes b, a^{\prime} \otimes b^{\prime}\right\rangle=(-1)^{\operatorname{dim} b \cdot \operatorname{dim} a^{\prime}}\left\langle a, a^{\prime}\right\rangle \cdot\left\langle b, b^{\prime}\right\rangle .
$$

Die Homomorphisme

$$
\psi^{*}: S^{*} \rightarrow S^{*} \otimes S^{*} \quad \text { und } \quad \psi_{*}: S_{*} \otimes S_{*} \rightarrow S_{*}
$$

sind per definitionem dual ( $\psi_{*}$ ist das Produkt im Ringe $\left.S_{*}\right)$. Also ist, wie man leicht überlegt,

$$
\left\langle\psi^{* *} u, a^{\prime} \otimes b^{\prime}\right\rangle=\left\langle u, \psi_{*}\left(a^{\prime} \otimes b^{\prime}\right)\right\rangle \quad \text { für } u \in S^{* *} .
$$

Wenn wir für $u$ einen Cohomologie-Automorphismus $\lambda$ einsetzen, dann folgt (Lemma 1.7 und (19), (20))

$$
\left\langle\lambda, a^{\prime} b^{\prime}\right\rangle=\left\langle\lambda, a^{\prime}\right\rangle \cdot\left\langle\lambda, b^{\prime}\right\rangle,
$$

wo $a^{\prime} b^{\prime}=\psi_{*}\left(a^{\prime} \otimes b^{\prime}\right)$ das Produkt in der Algebra $S_{*}$ bezeichnet. Nach MrLNOR [16] ist $S_{*}$ das Tensorprodukt der durch Unbestimmte $\tau_{0}, \tau_{1}, \tau_{2}, \ldots$ erzeugten Grassmannschen Algebra $\left(\tau_{i} \in S_{2 q^{i}-1}\right)$ und dem Polynomring in Unbestimmten $\xi_{1}, \xi_{2}, \ldots$ iiber $\boldsymbol{Z}_{q}$, wo $\xi_{i} \in S_{2 q^{i}-2}$. Wegen $S^{* *}=\operatorname{Hom}\left(S_{*}, \boldsymbol{Z}_{q}\right)$ und wegen (21) ist ein Cohomologie-Automorphismus durch die Werte $\left\langle\lambda, \xi_{i}\right\rangle$ und $\left\langle\lambda, \tau_{i}\right\rangle$ festgelegt $(\langle\lambda, 1\rangle=1)$. Nun betrachten wir mit Milnor den Linsenraum $X=\lim _{N \rightarrow \infty} S^{2 N+1} / \boldsymbol{Z}_{q}$ und die erzeugenden Elemente $\alpha \in H^{1}\left(X ; \boldsymbol{Z}_{q}\right)$ und $A \in H^{2}\left(X ; \boldsymbol{Z}_{q}\right)$. Dann folgt $[16$, Lemma 4 und 6$]$

$$
\lambda(\alpha)=\alpha+\sum_{i=0}^{\infty}\left\langle\lambda, \tau_{i}\right\rangle \beta^{q^{i}}
$$

und deshalb $\left\langle\lambda, \tau_{i}\right\rangle=0$, da $\operatorname{dim} \beta^{q^{t}}-\operatorname{dim} \alpha$ ungerade ist. Ebenso folgt

$$
\lambda(\beta)=\sum_{i=0}^{\infty}\left\langle\lambda, \xi_{i}\right\rangle \beta^{a^{i}}, \quad\left(\xi_{0}=1\right)
$$


Für die natürliche Projektion

$$
\tau: X \rightarrow \boldsymbol{P}_{\infty}(\boldsymbol{C})
$$

ist $\pi^{*} g=\beta$, wo $g$ ein geeignetes erzeugendes Element von $H^{2}\left(\boldsymbol{P}_{\infty}(\boldsymbol{C}) ; \boldsymbol{Z}_{q}\right)$ ist. Da $\pi^{* *}$ injektiv ist, folgt aus (23)

$$
\lambda(g)=g+\sum_{i=1}^{\infty}\left\langle\lambda, \xi_{i}\right\rangle g^{q^{i}} .
$$

Der Koeffizient $a_{j}$ der Potenzreihe $\lambda(x)$ in (16) ist also gleich $\left\langle\lambda, \xi_{j}\right\rangle$. Damit ist bewiesen, $\mathrm{daB} \operatorname{Coh}\left(\boldsymbol{Z}_{q}\right) \rightarrow G\left(\boldsymbol{Z}_{q}\right)$ injektiv ist.

Es bleibt zu zeigen, daß jede Potenzreihe

$$
\sum_{j=0}^{\infty} a_{j} x^{a^{i}} \in G\left(\boldsymbol{Z}_{q}\right), \quad\left(a_{0}=1\right),
$$

als Potenzreihe eines Cohomologie-Automorphismus auftritt. Wir definieren eine Element $\lambda \in S^{* *}$, indem wir das entsprechende Element $\lambda^{\prime} \in \operatorname{Hom}\left(S_{*}, Z_{q}\right)$ angebert. $\lambda^{\prime}$ ist durch folgende Angaben bestimmt.

a) $\lambda^{\prime}: S_{*} \rightarrow Z_{q}$ ist ein Ring-Homomorphismus,

b) $\lambda^{\prime}\left(\tau_{i}\right)=\left\langle\lambda, \tau_{i}\right\rangle=0, \lambda^{\prime}\left(\xi_{j}\right)=\left\langle\lambda, \xi_{j}\right\rangle=a_{j}$.

In $\S 4$ von [16] betrachtet MiLnor (für endliche $C W$-Komplexe $X$ ) einen RingHomomorphismus $\lambda^{*}$, den wir hier mit $\Lambda$ bezeichnen, um Verwechslungen zu vermeiden:

$$
A: H^{*}(X) \rightarrow H^{*}(X) \otimes S_{*} .
$$

Man kann $A$ ohne weiteres für einen beliebigen Raum $X$ definieren, wenn man $H^{*}(X)$ durch $H^{* *}(X)$ ersetzt. $\lambda^{\prime \prime}$ sei der Ring-Homomorphismus

$$
\lambda^{\prime \prime}: H^{*}(X) \otimes S_{*} \rightarrow H^{*}(X) \quad \text { mit } \quad \lambda^{\prime \prime}(a \otimes b)=a \cdot \lambda^{\prime} b .
$$

Dann ist nach [16, Lemma 4$]$

$$
\lambda=\lambda^{\prime \prime} \circ \Lambda .
$$

Also ist $\lambda: H^{*}(X) \rightarrow H^{*}(X)$ auch ein Ring-Homomorphismus und deshalb, wie man leicht sieht, ein Cohomologie-Automorphismus mit der gegebenen Potenzreihe (24).

Der Satz wird für $q=2$ fast genauso bewiesen (vgl. [16, Appendix 1]).

1.9. Für $q=2$ läßt sich jeder Cohomologie-Automorphismus $\lambda$ durch die Steenrodschen reduzierten Quadrate $S q^{i}$ ausdrücken, d.h. die $j$-dimensionale Komponente $\lambda_{j}(j=0,1,2, \ldots)$ ist ein "Polynom" in den $S q^{i}$. Dies ist klar, da die Steenrodsche Algebra durch die $S q^{i}$ erzeugt wird. Für ungerades $q$ wird die Steenrodsche Algebra durch die Bocksteinsche Operation $\delta$ und die Steenrodschen reduzierten Potenzen $\mathscr{P}^{\text {r }}$ (vgl. 1.1) erzeugt. Es gilt aber auch hier:

Lemma. Ein Cohomologie-Automorphismus $\lambda$ für die Cohomologie mit Koeffizienten in $\boldsymbol{Z}_{q}$ ( $q$ ungerade Primzahl) läßt sich durch die Steenrodschen reduzierten Potenzen ausdrücken, d.h. $\lambda_{j}(j=0,1,2, \ldots)$ ist ein Polynom. in den $\mathscr{P P}^{r}$, (oder 
anders gesagt: $\lambda_{i}$ gehört zu der durch die $\mathscr{P}^{*}$ erzeugten Unieralgebra $S t^{*}$ von $\left.S^{*}\right)$. Insbesondere ist $\lambda_{j}=0$, wenn $j=0(\bmod 2(q-1))$.

BEweis. $\lambda$ nimmt auf allen Elementen von $S_{*}$, die dem durch $\tau_{0}, \tau_{1}, \tau_{2}, \ldots$ erzeugten Ideal $I$ von $S_{*}$ angehören, den Wert 0 an (vgl. (22)). Aus (19) und (20) folgt, daß jedes Element von $S t^{*}$ das Ideal $I$ annuliert. Aus [16, Lemma 8] folgt umgekehrt, daß ein Element von $S^{*}$, das $I$ annuliert, zu $S t^{*}$ gehört.

1.10. Die Steenrodsche Algebra $S^{*}$ besitzt einen kanonischen Anti-Automorphismus [16, §7], der zu einem Anti-Automorphismus von $S^{* *}$ erweitert werden kann. Aus seiner Definition folgt unmittelbar, daß er einen Cohomologie-Automorphismus $\lambda$ in $\lambda^{-1}$ überführt.

\section{$\S 2$. Charakteristische Klassen}

2.1. Bezeichnungen. Wir betrachten im folgenden reelle VektorraumBündel, von denen wir durchweg voraussetzen, daB sie einen parakompakten Basisraum haben. Sie lassen dann die orthogonale Gruppe als Strukturgruppe $z u$, und es gibt immer Bündelabbildungen in das universelle Bündel [15]. In den jeweiligen Abschnitten wird die singuläre Cohomologie mit Koeffizienten in einen festen Primkörper der Charakteristik $q$ betrachtet, wobei auch $q=0$ vorkommen wird. Die Kaeffizientenbereiche werden also $\boldsymbol{Z}_{q}(q \neq 0)$ bzw. $\boldsymbol{Q}$. der $\mathrm{Körper} \mathrm{der} \mathrm{rationalen} Z$ ahlen sein, der auch mit $\boldsymbol{Z}_{0}$ bezeichnet werde. Bei der Anschreibung der Cohomologie-Gruppen wird der Koeffizientenbereich häufig weggelassen. Falls $q \neq 2$, wird immer vorausgesetzt, daß die auftretenden Vektorraum-Bündel orientiert sind, also die spezielle orthogonale Gruppe als Strukturgruppe zulassen.

Wir setzen die Theorie der charakteristischen Klassen als bekannt voraus $[5,11,15]$. Die Stiefel-Whitneyschen Klassen werden nur für $q=2$ betrachtet. Die Pontrjaginschen Klassen sind ganzzahlige Klassen. Wenn wir für die Cohomologie den Primkörper der Charakteristik $q$ verwenden, dann bezeichnet $p_{i}$ der Einfachheit wegen das Bild der ganzzahligen $4 i$-dimensionalen Pontrjaginschen Klasse unter dem Koeffizienten-Homomorphismus von $\boldsymbol{Z}$ in den Primkörper. Für $q=2$ ist also $p_{i}=w_{2}^{2}$, wo $w_{j}$ die $j$-dimensionale StiefelWhitneysche Klasse bezeichnet (vg1. [5, Part II, §30.8]).

2.2. Für die Cohomologie wird ein fester Primkörper $\boldsymbol{Z}_{q}(q$ beliebig) als Koeffizientenbereich verwandt. Gegeben sei ein reelles Vektorraum-Bündel $\xi$ mit Faser $\boldsymbol{R}^{n}$, Totalraum $E_{\xi}$, Basis $B_{\xi}$ und Projektion $\pi_{\xi}$. (Den Index $\xi$ werden wir häufig weglassen, wenn keine Verwechslungen zu befürchten sind.) Den Verabredungen von 2.1 folgend wird für $q \neq 2$ vorausgesetzt, daB $\xi$ orientiert ist. $E_{\xi, 0}=E_{0}$ bezeichnet wie bei Milnor [15] die Menge der Punkte von $E$, die nicht gleich der 0 ihrer Faser sind. Es gibt eine kanonische Klasse $U \in H^{n}\left(E, E_{0}\right)$ (vg1. z.B. [15, Chap. VII, Theorem 10, und Chap. VII, Theorem 10']) und den Thomschen (additiven) Isomorphismus

$$
\left\{\begin{array}{lll}
\Phi: H^{i}(B) \rightarrow H^{i+n}\left(E, E_{0}\right), & \Phi(x)=\pi^{*} x \cup U, & x \in H^{i}(B), \\
\Phi: H^{* *}(B) \rightarrow H^{* *}\left(E, E_{0}\right), & \Phi(x)=\pi^{* *} x \cup U, & x \in H^{* *}(B) .
\end{array}\right.
$$


Wenn $q \neq 0$, dann können wir einen Cohomologie-Automorphismus $\lambda$ betrachten $(1.1,1.3)$. Wir setzen

$$
\underline{\lambda}(\xi)=\Phi^{-1}(\lambda(U))=\Phi^{-1} \lambda \Phi(1) \in H^{* *}\left(B_{\xi}\right)
$$

Wir haben damit eine Operation $\underline{\lambda}$ definiert, welche jedem Vektorraum-Bündel ein Element des Cohomologie-Ringes $H^{* *}$ seiner Basis zuordnet. Um die Eigenschaften von $\underline{\lambda}$ besser formulieren zu können, führen wir folgende Definition ein.

2.3. Für die Cohomologie wird wieder ein fester Primkörper als Koeffizientenbereich verwandt. Wenn die Charakteristik verschieden von 2 ist, werden die Vektorraum-Bündel als orientiert vorausgesetzt.

DEFINITION. Eine Operation $\mu$, welche jedem Vektorraum-Bündel $\xi$ ein Element $\mu(\xi) \in H^{* *}\left(B_{\xi}\right)$ zuordnet, dessen 0-dimensionale Komponente gleich 1 ist, heißt multiplikativer Funktor, wenn gilt

(i) $\mu$.verhält sich natürlich bei Bündelabbildungen.

(ii) Für die Whitneysche Summe $\xi \oplus \eta$ zweier Vektorraum-Bündel $\xi, \eta$ mit $B_{\xi}=B_{\eta}$ ist

$$
\mu(\xi \oplus \eta)=\mu(\xi) \cdot \mu(\eta)
$$

Mit $G^{* *}(X)$ werde für $q=2$ die Menge aller Elemente von $H^{* *}(X)$ verstanden, deren 0-dimensionale Komponente gleich 1 ist. Für $q \neq 2$ sei $G^{* *}(X)$ dagegen die Menge aller Elemente von $H^{* *}(X)$, deren 0-dimensionale Komponente gleich 1 ist und deren ungerade-dimensionale Komponenten verschwinden. In beiden Fällen ist $G^{* *}(X)$ eine abelsche Gruppe bezüglich des Cüp-Produkts.

Aus der Theorie der klassifizierenden Räume folgt, daß sich $\mu(\xi)$ für $q=2$ durch die Stiefel-Whitneyschen Klassen und für $q \neq 2$ durch die Pontrjaginschen Klassen und die Eulersche Klasse ausdrücken läßt. Per definitionem ist $\mu(\xi) \in G^{* *}\left(B_{\xi}\right)$ für $q=2$. Aber auch für $q \neq 2$ ist dies richtig. Wie wir später sehen werden (2.5) kommt (fïr ungerades $q$ ) die Eulersche Klasse in Wirklichkeit nicht vor, so da $B$ die von 0 verschiedenen Komponenten von $\mu(\xi)$ sogar durch 4 teilbare Dimension haben.

2.4. In 2.2 hatten wir einen Cohomologie-Automorphismus $\lambda$ die Operation $\underline{\lambda}$ zugeordnet. Die 0 -dimensionale Komponente von $\underline{\lambda}$ ist gleich 1 , da $\lambda_{0}$ die Identität ist.

SaTz. Die Operation $\underline{\lambda}$ ist ein multiplikativer Funktor im Sinne der Definition 2.3.

Der. Beweis geht genauso wie bei MiLnor [15, Chap. VII (Verification of axioms)]. Siehe insbesondere Milnors Theorem.11, das die Eigenschaft 2.3 (ii) für den Cohomologie-Automorphismus' $S q$ betrifft. Beim Beweis hat man 1.3 (iii) zu verwenden. Vorzeichenschwierigkeiten treten nicht auf, da $\underline{\lambda}^{\prime} \xi$ ) mit jedem Element von $H^{* *}\left(B_{\xi}\right)$ vertauschbar ist. 
2.5. Ein multiplikativer Funktor im Sinne von 2.3 ist für Charakteristik $q=2$ die totale Stiefel-Whitneysche Klasse $w=\sum_{i=0}^{\infty} w_{i}\left(w_{0}=1, w_{i} \in H^{i}\right)$ und für Charakteristik $q \neq 2$ die totale Pontrjaginsche Klasse $p=\sum_{i=0}^{\infty} p_{i}\left(\dot{p}_{0}=1, p_{i} \in H^{4 i}\right)$, s. $[11, \S 4.5]$. Es sei $\left\{K_{j}\left(w_{1}, \ldots, w_{j}\right)\right\}$ eine multiplikative Folge von Polynomen mit Koeffizienten in $\boldsymbol{Z}_{2}(\mathrm{~s} .[11, \S 1])$. Die $\boldsymbol{w}_{i}$ werden hier als Unbestimmte über $\boldsymbol{Z}_{2}$ betrachtet. Ordnet man dann jedem Vektorraum-Bündel $\boldsymbol{\xi}$ das Element

$$
\sum_{j=0}^{\infty} K_{i}\left(w_{1}(\xi), \ldots, w_{j}(\xi)\right) \in H^{* *}\left(B_{\xi}\right)
$$

zu, dann erhält man einen multiplikativen Funktor im Sinne von 2.3.

Ebenso liefert jede multiplikative Folge $\left\{K_{j}\left(p_{1}, \ldots, p_{j}\right)\right\}$, von Polynomen mit Koeffizienten in $\boldsymbol{Z}_{q}(q \neq 2)$ einen multiplikativen Funktor, indem man jedem orientierten Vektorraum-Bündel $\xi$ das Element

$$
\sum_{j=0}^{\infty} K_{j}\left(p_{1}(\xi), \ldots, p_{j}(\xi)\right) \in H^{* *}\left(B_{\xi}\right)
$$

zuordnet.

Wir werden, zeigen, daß man auf diese. Weise alle multiplikativen Funktoren erhält:

Gegeben sei ein multiplikativer Funktor $\mu(q=2)$. Dann gibt es eine and nur eine Potenzreihe $Q(\mu, x) \in \boldsymbol{Z}_{2}[[x]]$, so daß $\mu(\xi)=Q\left(\mu, w_{1}(\xi)\right)$ für jedes Vektorraum-Bündel $\xi$ mit Faser $\boldsymbol{R}^{\mathbf{1}}$. Dies ergibt sich mit Hilfe des universellen Bündels (Faser $\boldsymbol{R}^{1}$ ) über dem unendlich-dimensionalen reellen projektiven Raum $\boldsymbol{P}_{\infty}(\boldsymbol{R})$. Zu dieser Potenzreihe gehört nun eine multiplikative Folge von Polynomen [1, §1] und zu dieser nach (3) ein multiplikativer Funktor, der mit $\mu$ übereinstimmt. Diese Ửbereinstimmung ist klar für Vektorraum-Bündel, die Whitneysche Summe von Bündeln mit Faser $\boldsymbol{R}^{1}$ sind, und deshalb für jedes Bündel $\xi$, da man zu $\xi$ (Faser $\boldsymbol{R}^{n}$ ) einen Raum $Y$ und eine stetige Abbildung $f: Y \rightarrow B_{\xi}$ finden kann, so daB $f^{*} \xi$ Whitneysche Summe von $n$ Bündeln mit Faser $\boldsymbol{R}^{1}$ über $Y$ ist und daß $f^{* *}: H^{* *}\left(B_{\xi}\right) \rightarrow H^{* *}(Y)$ injektiv ist. $Y$ ist der Totalraum des zu $\xi$ assoziierten Bündels mit $\boldsymbol{O}(n) /\left(\boldsymbol{Z}_{2}\right)^{n}$ als Faser [5, Part I, \$9.2]. ( $Y$ ist parakompakt als Faserbündel über einem parakompakten Raum mit kompakter Faser.)

Gegeben sei ein multiplikativer Funktor $\mu(q \neq 2)$. Dann gibt es eine und nur eine Potenzreihe $Q(\mu, z) \in \boldsymbol{Z}_{q}[[z]]$ mit $\mu(\xi)=Q\left(\mu, p_{1}(\xi)\right)$ für jedes orientierte Vektorraum-Bündel $\xi$ mit Faser $\boldsymbol{R}^{2}$. Dies ergibt sich, indem man beachtet, $\operatorname{da} \beta \mu(\xi)=\mu(\xi \oplus 1)$, wo 1 das triviale Vektortaum-Bündel mit Faser $\boldsymbol{R}^{\mathbf{1}}$ ist, und daß $H^{* *}\left(B_{\mathbf{S O}(3)}\right)=\grave{Z}_{q}\left[\left[p_{1}\right]\right]$. Zur Potenzreihe $Q(\mu, z)$ gehört eine multiplikative Folge von Polynomen und zu dieser nach (4) ein multiplikativer Funktor, der mit $\mu$ übereinstimmt. Diese Übereinstimmung ist zunächst klar für orientierte Vektorraum-Bündel, die Whitneysche Summe von orientierten Bündeln mit Faser $\boldsymbol{R}^{2}$ sind, und dann für jedes Bündel $\xi$, da man zu $\xi$ einen 
Raum $Y$ und eine stetige Abbildung $f: Y \rightarrow B_{\xi}$ finden $k a n n$, so da $B f^{*} \xi$ Whitneysche Summe von orientierten Vektorraum-Bündeln mit Faser $\boldsymbol{R}^{2}$ und eventuell einem trivialen Bündel mit Faser $\boldsymbol{R}^{\mathbf{1}}$ ist und daß $f^{* *}$ injektiv ist [5, Part $\mathrm{I}$, $\S 9.3]$.

\subsection{Die Überlegungen von 2.5 haben gezeigt:}

SaTz. Ordnet man jedem multiplikativen Funktor $\mu$ (s. 2.3) die multiplikative Folge von Polynomen mit der charakteristischen Potenzreihe $Q(\mu, x) \in \boldsymbol{Z}_{2}[[x]]$ bzw. $Q(\mu, z)=\boldsymbol{Z}_{q}[[z]](q \neq 2) z u$, dann erhält man eine eindentige Korrespondenz zwischen den multiplikativen Funktoren und den multiplikativen Folgen von Polynomen mitc Koeffizienten in $\boldsymbol{Z}_{q}$. Die Potenzreihe $Q(\mu, x)$ bzw. $Q(\mu, z)$ ist für $q=2$ bestimmt durch:

$$
\mu(\xi)=Q\left(\mu, w_{1}(\xi)\right) \quad \text { für jedes Vektorraum-Bündel } \xi \text { mit Faser } \boldsymbol{R}^{1}
$$

und für $q \neq 2$ durch

(6) $\mu(\xi)=Q\left(\mu, p_{1}(\xi)\right)$ für jedes orientierte Vektorraum-Bündel mit Faser $\boldsymbol{R}^{2}$.

Schreibt man formal

(7) $w(\xi)=1+w_{1}(\xi)+\cdots+w_{n}(\xi)=\prod_{j=1}^{N}\left(1+x_{j}\right), \quad\left(N \operatorname{gro}, \operatorname{dim} x_{j}=1\right)$,

- dann ist für $q=2$

Schreibt man formal

$$
\mu(\xi)=\prod_{j=1}^{N} Q\left(\mu, x_{j}\right)
$$

$$
p(\xi)=\sum_{i=0}^{n} p_{i}(\xi)=\prod_{j=1}^{N}\left(1+x_{j}^{2}\right), \quad\left(N \operatorname{groB} ; \operatorname{dim} x_{i}=2\right),
$$

dann ist für $q \neq 2$

$$
\mu(\xi)=\prod_{j=1}^{N} Q\left(\mu, x_{j}^{2}\right) .
$$

Für einen Raum $X$ haben wir in [3] die abelsche Gruppe $K \boldsymbol{O}(X)$ definiert: Man betrachte alle Vektorraum-Bündel mit Basis $X$. (Die Vektorraum-Bündel brauchen nicht orientierbar zu sein; es wird an dieser Stelle auch zugelassen, daß das Vektorraum-Bündel über verschiedenen Zusammenhangskomponenten von $X$ verschiedene Faserdimensionen hat.) $F(X)$ sei die freie abelsche Gruppe, die von den Isomorphieklassen aller dieser Vektorraum-Bündel erzeugt wird. Für jedes Tripel $t=\left(\xi, \xi^{\prime}, \xi^{\prime \prime}\right)$ von Isomorphieklassen mit $\xi=\xi^{\prime} \oplus \xi^{\prime \prime}$ bilden wir das Element $[t]=\xi-\xi^{\prime}-\xi^{\prime \prime} \in F(X)$. Die Gruppe $K \boldsymbol{O}(X)$ ist definiert als die Quotientengruppe von $F(X)$ modulo der Untergruppe, die von allen Elementen der Form $[t]$ erzeugt wird. Für die Definition analoger Grothendieckscher Gruppen im Rahmen der algebraischen Geometrie siehe [6].

LEMMA. Der Primkörper $\boldsymbol{Z}_{q}$ diene als Koeffizientenbereich für die Cohomologie. Jeder multiplikative Funktor $\mu$ im Sinne von 2.3 induziert auf natürliche Weise einen Homomorphismus

$$
\mu: K \boldsymbol{O}(X) \rightarrow G^{* *}(X)<H^{* *}(X) .
$$


Bewers. Für $q=2$ ist dies klar: $\mu$ ist auf allen Erzeugenden von $F(X)$ definiert. (Zu jeder Erzeugenden $\xi$ gibt es eine Darstellung von $X$ als disjunkte Vereinigung offener und abgeschlossener Teilmengen $X_{i}$, so daß die Beschränkung $\xi_{i}$ von $\xi$ auf $X_{i}$ konstante Faserdimension hat. Dann ist $\mu(\xi) \in H^{* *}(X)$ gleich dem „direkten Produkt" $\operatorname{der} \mu\left(\xi_{i}\right) \in H^{* *}\left(X_{i}\right)$.) Da $\mu(\xi)$ in der abelschen Gruppe $G^{* *}(X)$ liegt, ist $\mu$ auf $F(X)$ wohldefiniert und, da es auf allen Elementen der Form $[t]$ gleich dem Einselement von $G^{* *}(X)$ ist, auch auf $K \boldsymbol{O}(X)$.

Für ungerades $q$ ist $\mu$ nach den Verabredungen von 2.3 zunächst nur für orientierte Vektorraum-Bündel definiert. Da es sich jedoch durch die Pontrjaginschen Klassen ausdrücken läßt, die für nicht notwendigerweise orientierte Vektorraum-Bündel erklärt sind $[11, \S 4.5]$, ist $\mu$ auch für nicht notwendigerweise orientierte Bündel und damit für die Erzeugenden von $F(X)$ definiert. $\mu$ ist gleich dex 1 von $G^{* *}(X)$ auf allen Elementen der Form $[t] \in F(X)$.

Jeder multiplikative Funktor $\mu$ ist durch eine multiplikative Folge $\left\{K_{j}\left(w_{i}, \ldots, w_{j}\right)\right\}$ bzw. $\left\{K_{i}\left(p_{1}, \ldots, p_{j}\right)\right\}$ bestinmt. Es sei nun $B_{\boldsymbol{O}}=\lim _{n \rightarrow \infty} B_{\boldsymbol{O}(n)}$ der klassifizierende Raum der unendlich orthogonalen Gruppe. Es ist für $q=2$

und für $q \neq 2$

$$
H^{* *}\left(B_{\boldsymbol{O}} ; \boldsymbol{Z}_{2}\right)=\mathbb{Z}_{2}\left[\left[w_{1}, w_{2}, \ldots\right]\right]
$$

$$
H^{* *}\left(B_{\boldsymbol{o}} ; \boldsymbol{Z}_{q}\right)=\boldsymbol{Z}_{q}\left[\left[p_{1}, p_{2}, \ldots\right]\right],
$$

wo $w_{i}$ die universelle Stiefel-Whitneysche Klasse der Dimension $i$ und $p_{i}$ die universelle Pontrjaginsche Klasse der Dimension 4i. ist. Der multiplikative Funktor $\mu$ ist durch das Element $\sum_{j=0}^{\infty} K_{j}\left(w_{1}, \ldots, w_{j}\right)$ bzw. $\sum_{j=0}^{\infty} K_{j}\left(p_{1}, \ldots, p_{j}\right)$ von $H^{* *}\left(B_{\boldsymbol{O}} ; \boldsymbol{Z}_{q}\right)$ bestimmt. Wir werden deshalb gelegentlich einen multiplikativen Funktor $\mu$ einfach als Element von $H^{* *}\left(B_{\boldsymbol{o}} ; \boldsymbol{Z}_{q}\right)$ ansehen und $\mu \in H^{* *}\left(B_{\boldsymbol{o}} ; \boldsymbol{Z}_{q}\right)$. schreiben. Dieses Element von $H^{* *}\left(B_{\boldsymbol{O}} ; \boldsymbol{Z}_{q}\right)$ ist gewissermaßen der Wert von $\mu$ auf dem stabilen universellen reellen Vektorraum-Bündel.

Ein multiplikativer Funktor $\mu: K \boldsymbol{O}(X) \rightarrow G^{* *}(X)<H^{* *}\left(X ; Z_{q}\right)$ ordnet auch jedem komplexen Vektorraum-Bündel $\xi$ über $X$ ein Element $\mu(\xi) \in G^{* *}(X) z u$, da $\xi$ als reelles Vektorraum-Bündel aufgefaßt werden kann. Ist $q \neq 2$, also $\mu$ gegeben durch eine multiplikative Folge $\left\{K_{i}\left(p_{1}, \ldots, p_{j}\right)\right\}$, dann ist $\mu(\xi)$ durch eine multiplikative Folge in den Chernschen Klassen von $\xi$ darstellbar, die man erhält, indem man in $K_{i}\left(p_{1}, \ldots, p_{j}\right)$ die Pontrjaginschen Klassen durch bekannte Polynome in den Chernschen Klassen ersetzt [11, \$1.3]:

$$
\mu(\xi)=\sum_{j=0}^{\infty} \widetilde{K}_{j}\left(c_{1}(\xi), \ldots, c_{i}(\xi)\right) \quad\left(\text { mit } \widetilde{K}_{2 i+1}=0\right)
$$
Es sei nun $B_{\boldsymbol{U}}=\lim _{n \rightarrow \infty} B_{\boldsymbol{U}(n)}$ der klassifizierende Raum der unendlichen unitären
Gruppe. Es ist

$$
H^{* *}\left(B_{\boldsymbol{V}} ; \boldsymbol{Z}_{q}\right)=\boldsymbol{Z}_{q}\left[\left[c_{1}, c_{2}, \ldots\right]\right] .
$$


Wir können daher $\mu$ als Element von $H^{* *}\left(B_{\boldsymbol{U}} ; \boldsymbol{Z}_{q}\right)$ autfassen. Auf Grund der natürlichen Inklusion $\boldsymbol{U}<\boldsymbol{O}$ hat man den Homomorphismus

$$
H^{* *}\left(B_{\boldsymbol{o}} ; Z_{q}\right) \rightarrow H^{* *}\left(B_{\boldsymbol{U}} ; \boldsymbol{Z}_{q}\right)
$$

$\operatorname{der}\left(\right.$ für $q \neq 2$ ) injektiv ist und bei dem $\mu$ (aufgefaßt als Element von $H^{* *}\left(\boldsymbol{B}_{\boldsymbol{O}} ; \boldsymbol{Z}_{q}\right)$ ) in $\mu$ (aufgefaßt als Element von $\left.H^{* *}\left(B_{\boldsymbol{U}} ; \boldsymbol{Z}_{q}\right)\right)$ übergeht.

2.7. Satz. Gegeben sei ein Cohomologie-Automorphismus $\lambda$ für die Cohomologie mit Koeffizienten in $\mathbf{Z}_{q} . Z u \lambda$ gehört eine Potenzreihe (s. 1.6., (16), (17)). Mit Hilfe von $\lambda$ definiert man für Vektorraum-Bündel den multiplikativen Funktor $\mu=\underline{\lambda}$, der nach $\left(7^{\prime}\right)$ bzw. $\left(8^{\prime}\right)$ mit Hilfe einer Potenzreihe berechnet werden kann. Es ist

(9) $Q(\mu, x)=\lambda(x) / x$ für $q=2$. und $Q\left(\mu, x^{2}\right)=\lambda(x) / x$ für $q$ ungerade.

BEWEIS FÜR UNGERADES $q$. Die Gleichung (6) ist für ein orientiertes $\xi$ mit Faser $\boldsymbol{R}^{2}$ nachzuweisen. Nun ist $\Phi^{-1}(U \cup U)$ die Eulersche Klasse $e \in H^{2}\left(B_{\xi}\right)$. Wir betrachten die Injektion $i: E \rightarrow\left(E, E_{0}\right)$ (s. 2.2). Dann ist $i^{*} U=\pi^{*} e$ (s. [15, Chap. VIII, Theorem 12]) und deshalb

$$
\Phi\left(e^{k-1}\right)=U^{k}, \quad k=1,2, \ldots
$$

Da $U$ Reduktion einer 2-dimensionalen ganzzahligen Klasse ist, wird $\lambda(U)$ durch die Potenzreihe $\lambda(x)$ gegeben, indem man $x$ durch $U$ ersetzt. Alsq entsteht wegen (10) das Element $\underline{\lambda}(\xi)=\Phi^{-1} \lambda(U)$ aus der Potenzreihe $\lambda(x) / x$, indem man $x$ durch $e$ ersetzt. Da $e^{2}=p_{1}(\xi)$, ist der Beweis beendet. (Für $q=2$ schließt man ähnlich. Man hat dann Bündel mit Faser $\boldsymbol{R}^{1} z u$ betrachten. Es gilt wieder eine Gleichung (10) mit $e=w_{1}(\xi)$.)

2.8. Es sei $\mu$ ein multiplikativer Funktor (2.3) und $\lambda$ ein CohomologieAutomorphismus. Ordnet man dann jedem Vektorraum-Bündel $\xi$ das Element $\lambda(\mu(\xi)) \in H^{* *}\left(B_{\xi}\right) \mathrm{zu}$, dann erhält man den multiplikativen Funktor $\lambda \circ \mu$, der wiederum mit Hilfe einer Potenzreihe berechnet werden kann (2.6.). Es folgt

$$
Q(\lambda \circ \mu, x)=Q(\mu, \lambda(x)) \quad \text { für } q=2
$$

Diese Formeln sind für das Folgende wichtig, wenn $\mu$ durch $\underline{\lambda}$ und $\lambda$ durch $\lambda^{-1}$ ersetzt wird. Den multiplikativen Funktor $\lambda^{-1} \circ \underline{\lambda}$ bezeichnen wir mit Wu $(\lambda)$, siehe 2.11. Es gilt dann nach (9), (11) und (11') und

$$
Q\left(\lambda^{-1} \circ \underline{\lambda}, x\right)=x / \lambda^{-1}(x) \quad \text { für } q=2
$$

2.9. Es besteht ein Zusammenhang zwischen dem Satz von Riemann-Roch (Toddschen Polynomen) und Cohomologie-Operationen [10], den wir in dieser Arbeit genauer untersuchen wollen (vgl. Einleitung). Für diesen Zweck ist 
das Verhalten der für die multiplikative Folge der Toddschen Polynome charakteristischen Potenzreihe $x /\left(1-e^{-x}\right)$ modulo einer Primzahl $q$ von Bedeutung.

LemMa. Ersetzi man in der Potenzireihe $x /\left(1-e^{-x}\right)$ die Unbestimmte $x$ durch $f \cdot x$ mit $f=q^{1 ;(q-1)}$ (q Primzahl), so erhält man eine Potenzreihe in $x$, deren Koeffizienten von der Gestalt $f^{m} \cdot r$ sind, wo $m$ ganz und nicht negativ und $r$ eine rationale $Z$ ahl ist, deren Zähler und Nenner zu q teilerfremd sind. Die Reduktion von $f^{m} \cdot r$ modulo $q$ ist per definitionem ein Element von $\boldsymbol{Z}_{q}$, das verschwindet, wenn $m>0$. Also ist die Reduktion modulo $q$ der Potenzreihe $f x /\left(1-e^{-f x}\right)$ wohldefiniert. Es gilt mit $f=q^{1 /(q-1)}$

$$
f x /\left(1-e^{-f x}\right) \equiv 1+\left(x-x^{q}+x^{q^{3}}-\cdots+(-1)^{j} x^{q i}+\cdots\right)^{q-1} \bmod q .
$$

Beweis. Der Exponent von $q$ in $k$ ! ist kleiner oder gleich $\frac{k-1}{q-1}$ (vgl. z.B. [23, p. 99]). Er ist gleich diesem Quotienten dann und nur dann, wenn $k$ eine Potenz von $q$ ist. Wegen des Wilsonschen Satzes ist

$$
\left(q^{i}\right) ! / q^{e} \equiv(-1)^{e} \equiv(-1)^{j} \bmod q, \quad \text { wobei } \varepsilon=\left(q^{j}-1\right) /(q-1) .
$$

Daraus folgt

$$
\left(1-e^{-j x}\right) / f x \equiv \sum_{j=0}^{\infty}(-1)^{j} x^{q i-1} \text { modulo } q .
$$

Bezeichnen wir die rechte Seite von (14) mit $G(x)$, dann bleibt zum Beweis von (13) nur zu zeigen, daß in $\boldsymbol{Z}_{q}[[x]]$

$$
1 / G(x)=1+(x \cdot G(x))^{q-1} \quad \text { oder } \quad x=x \cdot G(x)+(x \cdot G(x))^{q} .
$$

Dies ist aber richtig, wegen $(a+b)^{q}=a^{q}+b^{q}$ modulo $q$.

Für eine ungerade Primzahl $q$ betrachteten wir weitere Potenzreihen, die im Sinne des Lemmas nach $q$ reduzierbar sind. Es gilt

$$
\left\{\begin{array}{c}
\frac{f x}{\operatorname{tgh} f x} \equiv \frac{(f x) / 2}{\sinh (f x) / 2} \equiv f x /\left(1-\epsilon^{-f x}\right) \text { modulo } q \\
\left(q \text { ungerade Primzahl, } f=q^{1 /(q-1)}\right) .
\end{array}\right.
$$

Diese Kongruenzen folgen aus den Gleichungen

$$
x+\frac{x}{\operatorname{tgh} x}=e^{x} \cdot \frac{x}{\sinh x}=2 x /\left(1-e^{-2 x}\right)
$$

unter Berücksichtigung von (13) und der Kongruenzen

$$
f x \equiv 0 \bmod q, \quad e^{t x} \equiv 1 \bmod q, \quad 2^{q^{i}-1} \equiv 1 \bmod q .
$$

2.10. Lemma. Es sei $\left\{K_{j}\left(c_{1}, \ldots, c_{j}\right)\right\}$ die multiplikative Folge von Polynomen in den Unbestimmten $c_{i}$ mit Koeffizienten in $\mathbf{Z}_{q}$ und der charakteristischen Potenzreihe $Q(x) \in \boldsymbol{Z}_{q}[[x]],(q$ beliebige Primzahl). Es sei a eine Potenz von $q$. Setzi man in $K_{j a}$ die Unbestimmte $c_{i}$ gleich 0 , wenn $i$ nicht durch a teilbar ist, dann erhält man ein Polynom $K_{j}^{\prime}\left(c_{a}, c_{\grave{a} a}, \ldots, c_{j a}\right)$. Es ist

$$
K_{i}^{\prime}\left(c_{a}, c_{2 a}, \ldots, c_{j a}\right)=K_{j}\left(c_{a}, c_{2 a}, \ldots, c_{j a}\right) .
$$


BEWEIS. Wir setzen $c_{i}^{\prime}=c_{i a}$. Dann ist $\left\{K_{i}^{\prime}\right\}$ die multiplikative Folge von Polynomen, die zur Potenzreihe $Q^{\prime}(x)=\sum_{j=0}^{\infty} K_{j}^{\prime}(1,0, \ldots, 0) x^{j}$ gehört. Es ist
$[11, \S 1.2]$

$$
Q^{\prime}\left(x^{a}\right)=K\left(1+x^{a}\right)=K\left((1+x)^{a}\right)=(K(1+x))^{a}=(Q(x))^{a}=Q\left(x^{a}\right) .
$$

Also ist $Q^{\prime}(x)=Q(x)$, was zu beweisen war.

2.11. Für einen Cohomologie-Automorphismus $\lambda$ haben wir den multiplikativen Funktor $\lambda^{-1} \circ \underline{\lambda}$ mit $W u(\lambda)$ bezeichnet, da er, wie sich in $\S 3$ herausstellen wird, in speziellen Fällen von WU WEN-TSUN betrachtet wurde. Für ein Vektorraum-Bündel $\xi$ schreiben wir statt $\mathrm{Wu}(\lambda)(\xi)$ auch $\mathrm{Wu}(\lambda, \xi)$. Wenn $\xi$ das Tangentialbündel einer differenzierbaren Mannigfaltigkeit $M$ ist, dann setzen wir $\mathrm{Wu}(\lambda, M)=\mathrm{Wu}(\lambda, \xi)$. Wir untersuchen nun zunächst im Falle der Charakteristik $q=2$ den multiplikativen Funktor $W u(S q)$ (vgl. 1.1) und seinen Zusammenhang mit den Toddschen Polynomen $T_{i}\left(c_{1}, \ldots, c_{i}\right)$, vgl. $[11, \S 1]$. Nach Lemma 2.9 ist $2^{i} T_{i}\left(c_{1}, \ldots, c_{i}\right)$ ein Polynom mit rationalen Koeffizienten, deren Nenner den Faktor 2 nicht enthalten. Deshalb definiert $2^{i} T_{i}$ bei Reduktion $\bmod 2$ ein Polynom mit Koeffizienten in $\boldsymbol{Z}_{2}$.

SATZ. Für ein Vektorraum-Bündel $\xi$ gilt

$$
\mathrm{Wu}(S q, \xi)=\sum_{i=0}^{\infty} 2^{i} T_{i}\left(w_{1}(\xi), \ldots, w_{i}(\xi)\right) \bmod 2
$$

Wenn die Stiefel-Whitneyschen Klassen $w_{2 j+1}(\xi)$ verschwinden, dann ist

$$
\mathrm{Wu}(S q, \xi)=\sum_{i=0}^{\infty} 2^{i} T_{i}\left(w_{2}(\xi), \ldots, w_{2 i}(\xi)\right), \bmod 2 .
$$

Wenn das Vektorraum-Bündel $\xi$ eine schwache komplexe Struktur zuläßt [5, Part III, §2], dann sind seine Chernschen Klassen $c_{i}(\xi) \in H^{2 i}\left(B_{\xi} ; \boldsymbol{Z}\right)$ definiert, und es gilt

$$
\mathrm{Wu}(S q, \xi)=\sum_{i=0}^{\infty} 2^{i} T_{i}\left(c_{1}(\xi), \ldots, c_{i}(\xi)\right) \bmod 2
$$

BeweIs. (17) ist wegen Lemma 2.10 (mit $q=a=2$ ) eine Folgerung aus (16). Für ein Vektorraum-Bündel $\xi$ mit schwaeher komplexer Struktur verschwinden die $w_{2 j+1}(\xi)$ und außerdem ist $c_{i}(\xi)=w_{2 i}(\xi) \bmod 2$. Deshalb ist (18) ein Korollar von (17). Zum Beweis von (16) ist wegen (12) und Lemma 2.9 nur zu zeigen, daß in $\boldsymbol{Z}_{\mathbf{2}}[[x]]$ die Gleichung gilt

$$
x / S q^{-1} x=1+\sum_{j=0}^{\infty} x^{j^{j}},
$$

wo $x$ als das von 0 verschiedene Element von $H^{1}\left(\boldsymbol{P}_{\infty}(\boldsymbol{R}) ; \boldsymbol{Z}_{2}\right)$ gedeutet werden kann. Wegen $S q x=x+x^{2}$ ist

$$
x=S q^{-1} x+\left(S q^{-1} x\right)^{2} .
$$


Macht man für $S q^{-1} x$ den Ansatz von $1.6(17)$, dann folgt aus vorstehender Gleichung, daB

und damit (19).

$$
S q^{-1} x=x+x^{2}+\cdots+x^{2}+\cdots
$$

Wir bemerken noch, daß die ungerade-dimensionalen Komponenten von $\mathrm{Wu}(S q, \xi)$ verschwinden, wenn $w_{1}(\xi)=0$, d.h. wenn $\xi$ orientierbar ist. Das liegt daran, daß das Polynom $T_{2 j+1}\left(c_{1}, \ldots, c_{2 j+1}\right)$ durch $c_{1}$ teilbar ist [11, $\S 1.7]$.

2.12. In Analogie zum vorhergehenden Abschnitt befassen wir uns nun für ungerade Charakteristik $q$ mit dem für orientierte Vektorraum-Bündel definierten multiplikativen Funktor $\mathrm{Wu}(\mathscr{P})$. Wir betrachten die multiplikativen Folgen $\left\{L_{j}\left(p_{1}, \ldots, p_{j}\right)\right\}$ bzw. $\left\{\hat{A_{j}}\left(p_{1}, \ldots, p_{j}\right)\right\}$ mit den charakteristischen Potenzreihen $z^{\frac{1}{2}} / \operatorname{tgh} z^{\frac{1}{2}} \mathrm{bzw}, z^{\frac{1}{2}} /\left(2 \sinh \left(z^{\frac{1}{2}} / 2\right)\right)$, vgl. $[11, \S 1]$. Das Polynom $\hat{A}_{j}$ steht zu dem in $[11, \S 1.6]$ definierten Polynom $A_{j}$ in der Beziehung $A_{j}=2^{4 j} \hat{A}_{j}$. Nach Lemma 2.9 und (15) sind $f^{2 j} L_{j}$ und $f^{2 j} \hat{A_{j}}$ (für $f=q^{1 /(q-1)}$ ) Polynome, deren Koeffizienten die Gestalt $f^{m} \cdot r$ haben ( $m$ ganz und nicht negativ; $r$ eine rationale $Z$ ahl, deren Zähler und Nenner zu $q$ teilerfremd sind). Die Polynome $f^{2 j} L_{j}$ und $f^{2 j} \hat{A}_{j}$ verschwinden modulo $q$, wenn $2 j$ nicht durch $q-1$ teilbar ist. $q^{[2 /(q-1)]} L_{j}$ und $q^{[2 j /(q-1)]} \widehat{A_{j}}$ sind Polynome mit rationalen Koeffizienten, die ganz sind bezüglich $q$ (d.h. $q$ nicht im Nenner enthalten). Wenn $2 j \equiv 0 \bmod q-1$, dann gilt

$$
q^{2 j /(q-1)} L_{j} \equiv q^{2 j /(q-1)} \hat{A}_{j} \equiv q^{2 j /(q-1)} A_{j} \bmod q
$$

SATz. Es sei q eine ungerade Primzahl und $\mathscr{P}$ der Steenrodsche CohomologieAutomorphismus (vgl. 1.1) für die Cohomologie mit Koeffizienten in $\boldsymbol{Z}_{q}$. Es sei $f=q^{1 /(q-1)}$. Für ein orientiertes Vektorraum-Bündel $\xi$ gilt

$$
\left\{\begin{aligned}
\mathrm{Wu}(\mathscr{P}, \xi) & =\sum_{j=0}^{\infty} f^{2 i} L_{j}\left(p_{1}(\xi), \ldots, p_{j}(\xi)\right) \\
& =\sum_{j=0}^{\infty} f^{2 i} \hat{A}_{j}\left(p_{1}(\xi), \ldots, p_{j}(\xi)\right) \text { modulo } q .
\end{aligned}\right.
$$

Die s-dimensionale Komponente von $\mathrm{Wu}(\mathscr{P}, \xi)$ verschwindet, wenn s nicht durch $2(q-1)$ teilbar ist.

BeweIs. Zum Beweis von (20) ist wegen Lemma 2.9, (12') und (15) nur zu zeigen, daß in $\boldsymbol{Z}_{q}[[x]]$ die Gleichung gilt

$$
x \mid \mathscr{P}^{-1} x=1+\left(x-x^{q}+x^{q^{2}}-\cdots+(-1)^{i} x^{q i}+\cdots\right)^{q-1},
$$

wo $x$ als ein von 0 verschiedenes Element von $H^{2}\left(\boldsymbol{P}_{\infty}(\boldsymbol{C}) ; \boldsymbol{Z}_{q}\right)$ gedeutet werden kann. Nun ist $\mathscr{P} x=x+x^{q}$ und deshalb

$$
x=\mathscr{P}^{-1} x+\left(\mathscr{P}^{-1} x\right)^{q} .
$$


Macht man für $\mathscr{P}^{-1} x$ den Ansatz von 1.6 (16), dann folgt aus vorstehender Gleichung sofort, daß

$$
\mathscr{P}^{-1} x=x-x^{q}+x^{q^{2}}+\cdots(-1)^{j} x^{q^{j}}+\cdots
$$

und damit (21).

2.13. Es sei $q$ eine beliebige Primzahl und $f=q^{1 /(q \rightarrow 1)}$. Nach Lemma 2.9 ist $f^{j} T_{j}\left(c_{1}, \ldots, c_{j}\right)$ ein Polynom, dessen Koeffizienten die Gestalt $f^{m} \cdot r$ haben ( $m$ ganz und nicht negativ; $r$ eine rationale Zahl, deren Zähler und Nenner zu $q$ teilerfremd sind). $f^{j} T_{j}$ verschwindet modulo $q$, wenn $j$ nicht durch $q-1$ teilbar ist. $q^{[j /(q-1)]} T_{j}$ ist ein Polynom mit rationalen Koeffizienten, die ganz sind bezüglich $q$. FaBt man $p_{1}, p_{2}, p_{3}, \ldots$ in bekannter Weise als Polynom in den Unbestimmten $c_{1}, c_{2}, \ldots$ auf $[11, \S 1.3]$, dann gilt für eine ungerade Primzahl $q$

$$
\sum_{j=0}^{\infty} f^{i} T_{j}\left(c_{1}, \ldots, c_{j}\right)=\sum_{j=0}^{\infty} f^{2 i} \widehat{A}_{j}\left(p_{1}, \ldots, p_{j}\right) \text { modulo } q .
$$

Dies folgt unmittelbar aus der zweiten Kongruenz von (15), vgl. auch [11, $\S 1.7$ (12)]. Aus (20) und (22) ergibt sich:

SATz. Wenn das Vektorraum-Bündel $\xi$ eine schwache komplexe Struktur zuläßt [5, Part III, §2] und $c_{i}(\xi) \in H^{2 i}\left(B_{\xi} ; \mathbf{Z}\right)$ die Chernschen Klassen sind, dann gilt für eine ungerade Primzahl $q$

$$
\mathrm{Wu}(\mathscr{P}, \xi)=\sum_{j=0}^{\infty} f^{j} \cdot T_{j}\left(c_{1}(\xi), \ldots, c_{j}(\xi)\right) \text { modulo } q .
$$

2.14. Bemerkung. Für ein Vektorraum-Bündel $\xi$ (vgl. 2.2) und einen Cohomologie-Automorphismus $\lambda$ kann man leicht nachweisen, daß $\underline{\lambda}(\xi) \in H^{* *}\left(B_{\xi}\right)$ eine Invariante des stabilen Faser-Homotopie-Typs von $\xi$ ist (vgl. z. B. M, F. AтIYAн, Thom complexes [Proc. Lond. Math. Soc. (3) 11, $291-310$ (1961)]). Für $q=2$ erhält man mit Hilfe von $\lambda=S q$, daß die totale Stiefel-Whitneysche Klasse $w(\xi)$ Invariante des stabilen Faser-Homotopie-Typs von $\xi$ ist. Für $q$ ungerade erhält man mit Hilfe von $\lambda=\mathscr{P}$, daß bei Anschreibung von $p(\xi)$ in der Form $2.6(8)$ die elementar-symmetrischen Funktionen in den $x_{j}^{q-\mathbf{1}}$ Invarianten des stabilen FaserHomotopie-Typs von $\xi$ sind (in der Cohomologie mit Koeffizienten $\mathbf{Z}_{q}$ ). Durch Verwendung eines allgemeinen $\lambda$ kommt man über dieses Resultat nicht hinaus, da $\lambda(x) / x$ eine Potenzreihe in $x^{q-1}$ ist. Auch die Anwendung irgendwelcher stabiler Cohomologie-Operationen auf die besprochenen Invarianten liefert nur Polynome in diesen Invarianten.

2.15. Lemma. Wie in 2.2 sei $\xi$ ein reelles Vektorraum-Bündel. $\lambda$ sei ein Cohomologie-Automorphismus. Für $z \in H^{* *}\left(B_{\xi}\right)$ gilt

BEWEIS.

$$
\Phi(\lambda(z) \cup \underline{\lambda}(\xi))=\lambda \Phi(z) .
$$

$$
\begin{aligned}
\Phi(\lambda(z) \cup \underline{\lambda(\xi))} & =\pi^{* *} \dot{\lambda}(z) \cup \pi^{* *} \underline{\lambda}(\xi) \cup U \\
& =\lambda \pi^{* *}(z) \cup \lambda U=\lambda\left(\pi^{* *} z \cup U\right)=\lambda \Phi(z) .
\end{aligned}
$$

Bei dieser Rèchnung wurde 1.3 (iii) benutzt. 
Wenn $\xi$ ein triviales Bündel ist, dann ist $\underline{\lambda}(\xi)=1$ und $\lambda$ und $\Phi$ sind in diesem Spezialfall vertauschbar. Dies entspricht der-Vertauschbarkeit von $\lambda$ mit $E$ (vgl. 1.3). Im allgemeinen sind $\lambda$ und $\Phi$ nicht vertauschbar. Man hat den „IMultiplikator" $\underline{\lambda}(\xi)$, der im nächsten Paragraphen eine wesentliche Rolle spielen wird.

Das Lemma gilt natürlich auch für komplexe Vektorraum-Bündel. Man fasse diese einfach als reelle Bündel auf.

\section{§3. Differenzierbare Mannigfaitigkeiten}

3.1. Ein Cohomologie-Automorphismus $\lambda: H^{* *}\left(X ; \boldsymbol{Z}_{q}\right) \rightarrow H^{* *}\left(X ; \boldsymbol{Z}_{q}\right)$ kann nach 1.3 angesehen werden als eine natürliche Transformation der singulären Cohomologie-Theorie mit Koeffizienten $\boldsymbol{Z}_{q}$ in sich. $\lambda$ respektiert die multiplikative Struktur. Die Graduierung wird jedoch vernachlässigt. Die Primzahl $q$ ist im folgenden immer festgewählt. Deshalb wird wieder das Symbol $\boldsymbol{Z}_{q}$ bei der Anschreibung der Cohomologie-Gruppen weggelassen. Alle Mannigfaltigkeiten werden als kompakt und differenzierbar vorausgesetzt. Für ungerades $q$ wird überdies angenommen, $\mathrm{da} B$ sie orientiert sind.

Es sei $X$ eine Mannigfaltigkeit. Dann hat man den kanonischen Poincaréschen Isomorphismus

$$
D_{X}: H^{*}(X) \rightarrow H_{*}(X),
$$

zu dessen Definition man für ungerades $q$ die Orientierung von $X$ benötigt. Wenn $n$ die Dimension von $X$ ist, dann ist $D_{X}$ folgendermaßen definiert:

$$
D_{X}(a)=a \cap h,
$$

wo $h \in H_{n}(X)$ für ungerades $q$ durch die Orientierung von $X$ bestimmt ist. Für $q=2$ ist $h$ direkte Summe der $h_{i}$, wo $h_{i}$ das von 0 verschiedene Element von $H^{n}\left(X_{i}\right)$ ist und $X_{i}$ die endlich vielen Zusammenhangskomponenten von $X$ durchläuft. Für eine stetige Abbildung $f: Y \rightarrow X$ von Mannigfaltigkeiten hat man den Gysinschen Homomorphismus

$$
f_{*}: H^{*}(Y) \rightarrow H^{*}(X), \quad f_{*}=D_{\bar{X}}^{-1} \circ f_{\#} \circ D_{Y},
$$

wo $f_{\#}: H_{*}(Y) \rightarrow H_{*}(X)$ der durch $f$ induzierte Homomorphismus der Homologiegruppen ist. Für $f: Y \rightarrow X$ sind also die beiden Homomorphismen

$$
f^{*}: H^{*}(X) \rightarrow H^{*}(Y), \quad f_{*}: H^{*}(Y) \rightarrow H^{*}(X),
$$

definiert. $f^{*}$ ist ein Ring-Homomorphismus, $f_{*}$ ein additiver Homomorphismus. Sie genügen folgender Beziehung

$$
f_{*}\left(f^{*} x \cup y\right)=x \cup f_{*}(y), \quad x \in H^{*}(X), \quad y \in H^{*}(Y) .
$$

Faßt man $H^{*}(X)$ und $H^{*}(Y)$ (vermöge $f^{*}$ ) als $H^{*}(X)$-Moduln auf, dann besagt (5), daß $f_{*}$ ein Homomorphismus von $H^{*}(X)$-Moduln ist. (5) folgt mit Hilfe von (2) aus der analogen Eigenschaft von $f_{\#}$ : Man beachte, da $B$ $H_{*}(X)$ und $H_{*}(Y)$ beide wegen des cap-Produkts $H^{*}(X)$-Moduln sind. (Für $H_{*}(Y)$ benutzt man $f^{*}$.) 
Der Cohomologie-Automorphismus $\lambda$ ist mit $f^{*}$ vertauschbar, jedoch im allgemeinen nicht mit $f_{*}$. Es gilt aber folgender Satz.

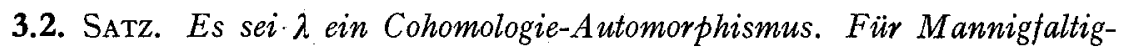
keiten $X$ ist dann das Element $\mathrm{Wu}\left(\lambda^{-1}, X\right) \in H^{*}(X)$ wohldefiniert (2.11). Es sei $f: Y \rightarrow X$ eine stetige Abbildung von Mannigfaltigkeiten. Dann ist

$$
f_{*}\left(\lambda y \cdot \mathrm{Wu}\left(\lambda^{-1}, Y\right)\right)=\lambda f_{*} y \cdot \mathrm{Wu}\left(\lambda^{-1}, X\right) \quad \text { für } y \in H^{*}(Y) .
$$

3.3. Bevor wir zum Beweis dieses Satzes kommen, sei auf seine Ähnlichkeit mit dem differenzierbaren Riemann-Rochschen Satz hingewiesen [3]. An Stelle von $\lambda$ hat man dort den Chernschen Charakter, der eine natürliche Transformation des Funktors $K^{*}(X)$ in $H^{* *}(X ; \boldsymbol{Q})$ liefert [4]. Man hat für eine stetige Abbildung $f: Y \rightarrow X$ von orientierten Mannigfaltigkeiten den Homomorphismus $f_{*}: H^{*}(Y ; \boldsymbol{Q}) \rightarrow H^{*}(X ; \boldsymbol{Q})$ und unter besonderen Voraussetzungen den recht schwierig zu definierenden Homomorphismus $f_{!}: K^{*}(Y) \rightarrow K^{*}(X)$. Immer wenn man eine natürliche Transformation von einer "Cohomologie-Theorie" (eventuell ohne Dimensionsaxiom) in eine andere hat, kann man nach einem Riemann-Rochschen Satz vom Typ 3.2 fragen. Beide Theorien sollten ein Analogon zur Poincaréschen Dualität erfüllen, damit in beiden Cohomologie-Theorien für stetige Abbildungen von Mannigfaltigkeiten die kovarianten Homomorphismen vorhanden sind, um deren Verträglichkeit (bzw. gerade Nicht-Verträglichkeit) mit der gegebenen natürlichen Transformation es sich ja handelt. Vielleicht werden wir an anderer Stelle auf dieses allgemeine Prinzip eingehen, das uns durch den Satz von Riemann-Roch im Sinne von GrothendiEck [6] nahegelegt wurde. Der Satz 3.2 stellt jedenfalls das einfachste Beispiel für Sätze dieses Typs dar, weil es sich um die gewöhnliche Cohomologie-Theorie und eine natürliche Transformation dieser Theorie in sich handelt.

3.4. Beweis von Satz 3.2. Das tangentielle Vektorraum-Bündel von $X$ bzw. $Y$ werde mit $\xi$ bzw. $\eta$ bezeichnet. Für $f: Y \rightarrow X$ betrachten wir das Element $\eta-f^{*} \xi \in K \boldsymbol{O}(Y)$ (s. 2.6), bezeichnen es mit $t f$ oder $t(f)$ und nennen es das Tangentialbündel von $f$. Wenn $f$ die Abbildung von $Y$ auf einen Punkt $X=\left\{x_{0}\right\}$ ist, dann ist das Tangentialbündel von $f$ gleich dem Tangentialbündel von $Y$ (aufgefaßt als Element von $K \boldsymbol{O}(Y)$ ). Wu $\left(\lambda^{-1}\right)$ ist ein multiplikativer Funktor im Sinne von 2.3, den wir für die Dauer des Beweises mit $\mu$ bezeichnen wollen: Die zu beweisende Gleichung (6) kann wegen (5) auch so geschrieben werden:

Es gilt nämlich

$$
f_{*}(\lambda y \cdot \mu(t f))=\lambda f_{*} y, \quad y \in H^{*}(Y) .
$$

$$
f_{*}(\lambda y \cdot \mu(\eta)) \cdot \mu(\xi)^{-1}=f_{*}\left(\lambda y \cdot \mu(\eta) \cdot \mu\left(f^{*} \xi\right)^{-1}\right)=f_{*}(\lambda y \cdot \mu(t f)) .
$$

Für Abbildungen $g: Z \rightarrow Y, f: Y \rightarrow X$ von Mannigfaltigkeiten $Z, Y, X$, deren Tangentialbündel mit $\zeta, \eta, \xi$ bezeichnet werden sollen, ist

$$
t(f \circ g)=\zeta-(f \circ g)^{*} \xi=g^{*}\left(\eta-f^{*} \xi\right)+\zeta-g^{*}(\eta)=g^{*} t(f)+t(g) .
$$


LEMMA. Wenn (7) für $f$ und g richtig ist, dann auch für fog.

Beweis. Es sei $z \in H^{*}(Z)$. Dann gilt nach (8)

$$
(f \circ g)_{*}(\lambda z \cdot \mu(t(f \circ g)))=f_{*} g_{*}\left(\lambda z \cdot g^{*} \mu(t f) \cdot \mu(t g)\right)
$$

Aus (5) und der Gleichung (7) für $g$ folgt

$$
f_{*} g_{*}\left(\lambda z \cdot g^{*} \mu(t f) \cdot \mu(t g)\right)=f_{*}\left(g_{*}(\lambda z \cdot \mu(t g)) \cdot \mu(t f)\right)=f_{*}\left(\lambda g_{*} z \cdot \mu(t f)\right) .
$$

Gleichung (7) für $f$ ergibt: $f_{*}\left(\lambda g_{*} z \cdot \mu(t f)\right)=\lambda f_{*} g_{*} z=\lambda(f \circ g)_{*} z$ und damit

$$
(f \circ g)_{*}(\lambda z \cdot \mu(t(f \circ g)))=\lambda(f \circ g)_{*} z, \quad \text { d.h. }(7) \text { für } f \circ g .
$$

Als nächstes bemerken wir, daß es genügt (7) für differenzierbare Abbildungen $\mathrm{zu}$ beweisen, da jede stetige Abbildung $f: Y \rightarrow X$ einer differenzierbaren homotop ist. Jede differenzierbare Abbildung $f: Y \rightarrow X$ läBt sich in eine differenzierbare Einbettung und eine Projektion mit einer Sphäre als Faser aufspalten: Es sei $i$ eine Einbettung von $Y$ in eine Sphäre $\mathbf{S}^{m}$ geeigneter Dimension $m$. Dann ist $(f, i)$ eine Einbettung $j$ von $Y$ in $X \times S^{m}$ :

$$
j=(f, i): Y \rightarrow X \times \boldsymbol{S}^{m}, \quad j(y)=(f(y), i(y)) .
$$

$\pi_{1}$ sei die Projektion von $X \times \boldsymbol{S}^{m}$ auf $X$, dann ist $f=\pi_{1} \circ j$. Wegen des Lemmas braucht (7) nur für Einbettungen und für die Projektion $\pi_{1}: X \times S^{m b} \rightarrow X$ bewiesen zu werden. Wir untersuchen zunächst den Fall der Projektion. Es sei $\pi_{2}$ die Projektion von $X \times \boldsymbol{S}^{m} \rightarrow \boldsymbol{S}^{m}$, ferner $\sigma$ das Tangentialbündel von $S^{m}$ und $\xi$ das von $X$. Es ist

$$
t\left(\pi_{1}\right)=\pi_{1}^{*} \xi+\pi_{2}^{*} \sigma-\pi_{1}^{*} \xi=\pi_{2}^{*} \sigma .
$$

Nun repräsentiert $\sigma$ in $K \boldsymbol{O}\left(\mathbf{S}^{m}\right)$ das Element $m \cdot 1$. Deshalb ist $\mu(\sigma)=1$ und ebenfalls $\mu\left(t\left(\pi_{1}\right)\right)=1$. Also besagt (7) für $f=\pi_{1}$

$$
\left(\pi_{1}\right)_{*} \lambda y=\lambda\left(\pi_{1}\right)_{*} y, \quad y \in H^{*}\left(X \times \mathbf{S}^{* *}\right) \text {. }
$$

Es sei g das zur Orientierung von $\mathbf{S}^{m}$ gehörige erzeugende Element von $H^{m}\left(\boldsymbol{S}^{m}\right)$. Jedes Element $y$ von $H^{*}\left(X \times \mathbf{S}^{m}\right)$ läßt sich dann auf eine und nur eine Weise in der Form $\pi_{1}^{*} a+\left(\pi_{1}^{*} b\right) \cdot\left(\pi_{2}^{*} g\right)$ schreiben $\left(a, b \in H^{*}(X)\right)$ und es ist $\left(\pi_{1}\right)_{*} y=b$. Da $\lambda y=\pi_{1}^{*} \lambda a+\left(\pi_{1}^{*} \lambda b\right)\left(\pi_{2}^{*} g\right)$ wegen $\lambda g=g$, folgt sofort (10). - Wir kommen nun zum Beweis von (7) füx den Fall einer Einbettung. $Y$ sei also eine differenzierbare Untermannigfaltigkeit von $X$ und $f: Y \rightarrow X$ die Injektion. Es sei $\nu$ das Normalbündel von $Y$ in $X$, dann gilt in $K \boldsymbol{O}(Y)$

$$
t(f)=-\nu \text {. }
$$

Es sei $E$ der Totalraum des Vektorraum-Bündels $v$. Mit Hilfe einer schlauchartigen Umgebung von $Y$ in $X$ (tubular neighborhood), die zu einer Umgebung des Nullschnittes von $v$ in $E$ homöomorph ist, konstruiert man durch geeignete Ausschneidung einen Isomorphismus

$$
\varepsilon: H^{*}\left(E, E_{0}\right) \rightarrow H^{*}(X, X-Y) .
$$


Ferner hat man den kanonischen Homomorphismus

$$
\varrho: H^{*}(X, X-Y) \rightarrow H^{*}(X) \text {. }
$$

In 2:2 wurde der Homomorphismus $\Phi$ betrachtet:

$$
\Phi: H^{*}(Y) \rightarrow H^{*}\left(E, E_{0}\right),
$$

der im Falle ungerader Charakteristik erst definiert ist, nachdem man dem Normalbündel eine Orientierung gegeben hat. Man kann bekanntlich $v$ so orientieren, daB

$$
f_{*}=\varrho \circ \varepsilon \text { ठ } \Phi
$$

Da der Cohomologie-Automorphismus $\lambda$ mit $\varrho$ und $\varepsilon$ vertauschbar ist, ergibt sich

$$
\lambda f_{*} y=\varrho \varepsilon \lambda \Phi y, \quad y \in H^{*}(Y)
$$

Aus 2.15 folgt nun unter Verwendung von (11) und (12), daß

$$
\lambda f_{*} y=\varrho \varepsilon \Phi(\lambda y \cdot \underline{\lambda} v)=f_{*}\left(\lambda y \cdot(\underline{\lambda}(t f))^{-1}\right) .
$$

Bisher wurde im Beweis nicht verwendet, $\operatorname{da} \beta \mu$ in (7) gleich $\mathrm{Wu}\left(\lambda^{-1}\right)$. Damit (14) in (7) übergeht, muß dies jetzt verwendet werden. Nun ist aber $\mathrm{Wu}\left(\lambda^{-1}\right)$ der multiplikative Funktor, welcher jedem Vektorraum-Bündel $\xi$ die Cohomologie-Klasse $(\underline{\lambda \xi})^{-1}$ zuordnet, da $(\lambda x / x)^{-1}=x /\left(\lambda^{-1}\right)^{-1} x$ (vgl. 2.7 (9) und $\left.2.8(12),\left(12^{\prime}\right)\right)$.

Der Beweis des Satzes 3.2 verlief analog zum Beweis des differenzierbaren Riemann-Rochschen Satzes [13].

3.5. Wir wollen Satz 3.2 jetzt auf den Fall anwenden, daß $X$ der einpunktige Raum $X=\left\{x_{\mathbf{0}}\right\}$ und $f$ die konstante Abbildung ist. In diesem Fall ist natürlich $\mathrm{Wu}\left(\lambda^{-1}, X\right)=1$. ' $\mathrm{Da} \lambda$ auf der Cohomologie des Punktes die Identität ist, geht die rechte Seite von (6) in $f_{*} y$ über. Für jedes $y \in H^{*}(Y)$ ist aber

$$
f_{*} y=y[Y] \cdot 1, \quad, 1 \in H^{0}(X),
$$

wo $y[Y]$ den Wert von $y$ auf dem (eventuell orientierten) Grundzyklus von $Y$ andeutet. Dabei nehmen alle Komponenten von $y$, deren Dimension nicht gleich der von $Y$ ist, den Wert 0 an. Satz 3.2 geht also in dem von uns betrachteten Spezialfall über in

$$
\left(\lambda y \cdot \mathrm{Wu}\left(\lambda^{-1}, Y\right)\right)[Y]=y[Y] \text {. }
$$

Ersetzt man in (16) zunächst $\lambda$ durch $\lambda^{-1}$ und dann $y$ durch $\lambda y$, dann folgt

$$
(\lambda y)[Y]=(y \cdot \mathrm{Wu}(\lambda, Y))[Y], \quad y \in H^{*}(Y) .
$$

Die Formel (17) motiviert, warum wir den multiplikativen Funktor $\lambda^{-1} \circ \underline{\lambda}$ mit $\mathrm{Wu}(\lambda)$ bezeichnet haben (2.11): Die Formel (17) stammt nämlich für $\lambda=S q$ oder $\lambda=\mathscr{P}$ von Wu Wen-Tsun $[25,26]$, der sie allerdings mit der Diagonalmethode bewiesen hat, auf die auch ADAMs [1] zurückgreift und auf 
die ebenfalls in [10] Bezug genommen wurde, während wir die differenzierbare Einbettung von $Y$ in ęine Sphäre und das entsprechende Normalbündel benutzt haben. Die Cohomologie-Klasse $W u(\lambda, Y)$ läßt șich mit Hilfe der Stiefel-Whitneyschen bzw. 'der Pontrjaginschen Klassen von $Y$ ausdrücken (Satz 2.6 und $2.8(12) ;\left(12^{\prime}\right)$ ). Für $\lambda=S q$ oder $\lambda=\mathscr{P}$ steht $W u(\lambda, Y)$ in enger Beziehung zu den Toddschen Polynomen $T_{i}\left(w_{1}(Y), \ldots, w_{i}(Y)\right)$ bzw. den $L$ Polynomen $L_{j}\left(p_{1}(Y), \ldots, p_{j}(Y)\right)$. Die Einzelheiten sind aus den Sätzen 2.11, $2.12,2.13 \mathrm{zu}$ entnehmen. Die in [10] ohne ausführlichen Beweis angegebenen Theoreme 2.1 bis 2.4 sind also in der vorliegenden Arbeit vollständig bewiesen.

3.6. Aus (17) folgen Relationen zwischen den Stiefel-Whitneyschen bzw. den Pontrjaginschen Zahlen einer Mannigfaltigkeit, wenn man für $\lambda$ beliebige Cohomologie-Automorphismen einsetzt und $y$ Polynome in den StiefelWhitneyschen bzw. den Pontrjaginschen Klassen der Mannigfaltigkeit durchläuft. In $\$ 5$ werden wir sehen, $\mathrm{da} \beta$ sich diese Relationen bereits erhalten lassen, wenn man (17) nür für $\lambda=S q$ bzw. $\lambda \doteq \mathscr{P}$ anwendet. Für die praktische Rechnung ist es jedoch bequemer, beliebige Cohomologie-Automorphismen heranzuziehen. Setzt man in (17) für $y$ das Einselement von $H^{*}(Y)$, dann ergibt sich

Sarz. Es sei $\lambda$ ein Cohomologie-Automorphismus für die Cohomologie mit Koeffizienten in $\boldsymbol{Z}_{q}$ ( $q$ beliebige Primzahl). Dann gilt für eine kompakte differenzierbare positiv-dimensionale Mannigfaltigkeit $Y$, die für ungerades $q$ unseren Verabredungen gemäß als orientiert vorausgesetzt wird,

$$
\mathrm{Wu}(\lambda, Y)[Y]=0 .
$$

Für eine kompakte orientierte differenzierbare Mannigfaltigkeit $M$ der Dimension $4 k$ spielt in der Cobordisme-Theorie die ganze Zahl $s(M)$ eine Rolle (vgl. z. B. [11, §6.3]), Schreibt man die ganzzahtigen Pontrjaginschen Klassen $p_{i}(M)$ formal in der Gestalt $2.6(8)$, dann ist

$$
s(M)=\left(\sum_{j=1}^{N} x_{i}^{2 k}\right)[M] .
$$

Wir geben nun einen direkten Beweis für einen Satz ton MILnor [17], den MiLnor auf dem Wege über Untersuchungen der Cobordisme-Algebra erhalten hat.

SATZ. Es sei $M$ eine kompakte orientierte differenzierbare Mannigfaltigkeit der Dimension 4k. Wenn $2 k+1$ eine Potenz der Primzahl $q$ ist, dann ist $s(M)$ durch q teilbar.

Bewsis. Wir setzen für den Moment $a=2 k+1$. Die Zahl $a$ ist eine Potenz der ungeraden Primzahl $q$. Nach Satz 1.8 gibt es einen Cohomologie-Automorphismus $\lambda$ für die Cohomologie mit Koeffizienten in $\boldsymbol{Z}_{q}$, für $\operatorname{den} \lambda^{-1} x=x-x^{a}$. Dann ist

$$
x / \lambda^{-1} x=1+x^{a-1}+\cdots+x^{j(a-1)}+\cdots \cdot
$$


Șchreibt man nun in der Cohomologie mit Koeffizienten in $\boldsymbol{Z}_{q}$ die Pontrjaginsche Klasse formal in der Gestalt $2.6(8)$, dann gilt $\left(2.8\left(12^{\prime}\right)\right)$

$$
\mathrm{Wu}(\lambda, M)=\prod_{j=1}^{N} x_{j} / \lambda^{-1} x_{j}
$$

Da $a-1=2 k=\operatorname{dim}(M) / 2$, ist $\mathrm{Wu}(\lambda, M)[M]=s(M) \bmod q$. Das beendet den Beweis wegen (18).

3.7. In $[11, \S 1.7]$ wurde ohne Beweis folgendes Lemma angegeben.

Lemma. Das Toddsche Polynom kann in eindeutiger Weise als Polynom mit ganzzahligen teilerfremden Koeffizienten dividiert durch eine positive ganze Zahl $\mu\left(T_{k}\right)$ geschrieben werden. Man hat

(19) $\mu\left(T_{k}\right)=\prod q^{[k /(q-1)]} \quad$ (Produkt über alle Primzahlen $q$ mit $\left.2 \leqq q \leqq k+1\right)$.

Wir tragen den Beweis hier nach. Abgesehen von der expliziten Formel (19) ist die Behauptung klar, da $T_{k}$ für $c_{i}=\left(\begin{array}{c}k+1 \\ i\end{array}\right)$ den. Wert 1 annimmt. Man beweist zunächst, $\mathrm{daB} \mu\left(T_{k+1}\right)$ ein Vielfaches von $\mu\left(T_{k}\right)$ ist. Dazu betrachtet man den Ring $\boldsymbol{Q}\left[c_{1}, d_{1}, c_{2}, c_{3}, \ldots\right]$ modulo dem von $d_{1}^{2}$ und den Monomen in den $c_{i}$ vom Gewichte größer als $k$ erzeugten Ideal $J$. Definiert man $c_{i}^{\prime}$ durch

$$
1+c_{1}^{\prime} x+c_{2}^{\prime} x^{2}+\cdots=\left(1+c_{1} x+c_{2} x^{2}+\cdots\right)\left(1+2 d_{1} x\right),
$$

dann ist wegen der Multiplikativität der Folge $\left\{T_{j}\right\}$ und wegen $T_{1}\left(c_{1}\right)=\frac{c_{1}}{2}$

$$
T_{k+1}\left(c_{1}^{\prime}, \ldots, c_{k+1}^{\prime}\right) \equiv d_{1} \cdot T_{k}\left(c_{1}, \ldots, c_{k}\right) \text { modulo } J \text {. }
$$

Daraus folgt in der Tat $\mu\left(T_{k+1}\right) \equiv 0 \bmod \mu\left(T_{k}\right)$. Nach den Berrerkungen über $f T_{j}$ zu Anfang von 2.13 genügt es zum Beweis von (19) zu zeigen, daß $f^{j} T_{j} \equiv \emptyset \bmod q$ für $j \equiv 0 \bmod q-1$. Es sei $j=r(q-1)$. Im komplexen projektiven Raum $\boldsymbol{P}_{r q}(\boldsymbol{C})$ mit $x \in H^{2}\left(\boldsymbol{P}_{r q}(\boldsymbol{C}) ; \boldsymbol{Z}_{q}\right)$ und $x \neq 0$ ist $\mathscr{P}\left(x^{r}\right)=x^{r}\left(1+x^{q-1}\right)^{r}$ und deshalb

$$
\mathscr{P}\left(x^{r}\right)\left[\boldsymbol{P}_{q}(\boldsymbol{C})\right]+0
$$

(Für $q=2$ setze $\operatorname{man} \mathscr{P}=S q$ ). Also ist die $2 r(q-1)$-dimensionale Komponente von $\mathrm{Wu}\left(\mathscr{P}, \boldsymbol{P}_{q r}(\boldsymbol{C})\right)$ nicht null $(3.5(17))$ und deshalb ist $q^{r} T_{r(q-1)} \neq 0 \bmod q$ (s. 2.13 (23) und 2.11 (18)). Ebenso beweist man entsprechende Aussagen über die Polynome $L_{k}$ und $A_{k}$ (s. [11, §1.5 und 1.6]).

3.8. Wir beschließen diesen Paragraphen mit Bemerküngen über kompakte nicht notwendigerweise differenzierbare Mannigfaltigkeiten. Sei $M$ eine solche Mannigfaltigkeit. $\lambda$ sei ein Cohomologie-Automorphismus für die Cohomologic mit Koeffizienten in $\boldsymbol{Z}_{2}$. Wegen des Poincaréschen Dualitätssatzes gibt es. dann genau ein Element $u$ von $H^{*}(M)$, so daß

$$
(\lambda y)[M]=(y \cdot u)[M] \quad \text { für alle } y \in H^{*}(M)
$$


Wir bezeichnen $u$ mit $\mathrm{Wu}(\lambda, M)$. Wegen 3.5 (17) stimmt dies mit unseren für differenzierbare Mannigfaltigkeiten eingeführten Bezeichnungen überein.

FraGe. Gilt unter der oben angegebenen Definition von $\mathrm{Wu}(\lambda, M)$ der Satz 3.2 für stetige Abbildungen $Y \rightarrow X$ kompakter nicht notwendigerweise differenzierbarer Mannigfaltigkeiten?

Für die Cohomologie mit Koeffizienten $\boldsymbol{Z}_{q}$ ( $q$ ungerade Primzahl) und für orientierte Mannigfaltigkeiten kann man ebenso die Klasse $W u(\lambda, M)$ einführen und die analoge Frage stellen.

Wir stellen noch fest, daB $\mathrm{Wu}(\lambda, M)$ per definitionem (20) eine Invariante des Homotopietyps von $M$ ist, ebenso natürlich $\lambda(\mathrm{Wu}(\lambda, M))$. Für differenzierbares $X$ ist $\lambda(\mathrm{Wu}(\lambda, X))=\underline{\lambda}(\xi)$, wo $\xi$ das Tangentialbündel von $X$ ist. Die Homotopieinvarianz von $\underline{\lambda}(\xi)$ (oder gleichwertig von $W u(\lambda, X)$ ) innerhalb der Kategorie der differenzierbaren Mannigfaltigkeiten folgt auch aus 2.14 und der Tatsache, daß homotopie-äquivalente differenzierbare Mannigfaltigkeiten Tangentialbündel haben, die vom gleichen stabilen Faser-HomotopieTyp sind (M. F. ATIYAH, Thom complexes (loc. cit. in 2.14), Theorem 3.6).

Legen wir nochmals $\boldsymbol{Z}_{2}$ als Koeffizientenbereich zugrunde. Für eine kompakte differenzierbare Mannigfaltigkeit $X$ ist $S q(\mathrm{Wu}(S q, X))$ gleich der totalen Stiefel-Whitneyschen Klasse von $X$. Für eine kompakte topologische Mannigfaltigkeit $M$ kann die Stiefel-Whitneysche Klasse $\tau_{i}(M)$ durch die Gleichung

$$
w(M)=\sum_{i=0}^{\infty} w_{i}(M)=S q((\mathrm{Wu}(S q, M))
$$

definiert werden. $w_{i}(M)$ ist ẹine Homotopieinvariante. Für eine kompakte differenzierbare Mannigfaltig̀keit $X$ ist $\mathrm{Wu}(\lambda, X)$ nach 2.8 ein gewisses Polynom in den $x_{i}(X)$. Es entsteht die Frage, ob sich $W u(\lambda, M)$ für eine kompakte topologische Mannigfaltigkeit $M$ durch das gleiche Polynom mittels der $w_{i}(M)$ ausdrücken läßt. Diese Frage wurde von ADAMS [1] bejaht.

Analoges gilt für. ungerades $q$. Statt $S q$ wählt man $\mathscr{P}$, und an Stelle der Stiefel-Whitneyschen Klassen treten die elementar-symmetrischen Funktionen in den $x_{j}^{q-1}$ (s. 2.14).

\section{$\S 4$. Der Satz von Adams}

4.1. Es sei $X$ ein endlicher $C W$-Komplex, dessen ganzzahlige Cohomologie keine Torsion habe. $H^{*}(X ; \boldsymbol{Z})$ läßt sich dann vermöge des KoeffizientenHomomorphismus $\boldsymbol{Z} \rightarrow \boldsymbol{Q}$ als Unterring vion $H^{*}(X ; \boldsymbol{Q})$ auffassen. Ein Element von $H^{*}(X ; \boldsymbol{Q})$ heißt ganz, wenn es zu $H^{*}(X ; \boldsymbol{Z})$ gehört. Ein Element $a$ von $H^{*}(X ; \boldsymbol{Q})$ heißt ganz bezüglich der Primzahl $q$, wenn es eine ganze zu $q$ teilerfremde Zahl $m$ gibt, so daß ma ganz ist. Der Homomorphismus

$$
\varrho_{q}: H^{*}(X ; \boldsymbol{Z}) \rightarrow H^{*}\left(X ; \boldsymbol{Z}_{q}\right)
$$

sei induziert durch $\boldsymbol{Z} \rightarrow \boldsymbol{Z}_{q}$. Dieser Homomorphismus kann erweitert werden auf den Unterring der bezüglich $q$ ganzen Elemente von $H^{*}(X ; \boldsymbol{Q})$ : Für $a \in H^{*}(X ; \boldsymbol{Q})$, das ganz bezüglich $q$ ist, definiert man $\varrho_{q}(a) \in H^{*}(X ; \boldsymbol{Q})$ durch 
die Gleichung

$$
\varrho_{q}(a)=m^{-1} \cdot \varrho_{q}(m a), \quad(m, q)=1, \quad m a \text { ganz. }
$$

$\varrho_{q}(a)$ hängt nicht von der Wahl von $m$ ab. (In (1) ist $m^{-1}$ natürlich im Körper $\mathbf{Z}_{q}$ zu berechnen.)

Für $X$ ist der Ring $K^{*}(X)$ definiert [4], der über $\boldsymbol{Z}_{2}$ graduiert ist:

$$
K^{*}(X)=K^{0}(X)+K^{1}(X) \text {. }
$$

$K^{0}(X)=K(X)$ wird mit Hilfe der komplexen Vektorraum-Bündel mit der Basis $X$ auf die Weise eingeführt, an die wir für reelle Vektorraum-Bündel in 2.6 erinnert hatten. Der Chernsche Charakter ist ein Ring-Homomorphismus

$$
c h: K^{*}(X) \rightarrow H^{*}(X ; \boldsymbol{Q}), \quad \text { s. }[4] \text {. }
$$

Wegen unserer Voraussetzung, daß $X$ torsionsfrei ist, existiert zu jedem Element $a \in H^{n}(X ; \boldsymbol{Z})$ ein Element $\xi \in K^{*}(X)$, dessen Chernscher Charakter mit $a$ beginnt, d.h.

$$
\operatorname{ch}(\xi)=a+\sum_{k=1}^{\infty} a_{n+2 k}, \quad a_{n+2 k} \in H^{n+2 k}(X ; \boldsymbol{Q}) .
$$

Die Elemente $a_{n+2 k}$ hängen im allgemeinen nicht nur von $a$ sondern auch von der Wahl von $\xi$ ab. Der folgende Satz von Adams [2] zeigt, daß in gewisser Weise die Reduktion der $a_{n+2}$ modulo $q$ invariant ist.

4.2. Satz (Adams). Es sei $X$ ein endicher $C W-K o m p l e x$, dessen ganzzahlige Cohomologie keine Torsion habe. $\xi$ sei Element von $K^{*}(X)$, dessen Chernscher Charakter von der Form (3) ist $\left(a \in H^{n}(X ; Z)\right)$. Dann ist $a_{n+2 k} \cdot q^{[k /(q-1)]}$ (für eine beliebige Primzahl $q$ ) ganz bezüglich $q$. Wenn $k$ nicht durch $q-1$ teilbar ist, und also $k /(q-1)>[k /(q-1)]$ ist, dann werde das Element $a_{n+2} \cdot q^{k /(q-1)}$ von $H^{n+2 k}(X ; \boldsymbol{R})$ betrachtet und $\varrho_{q}$ von diesem Element gleich 0 gesetzt. Damit ist $\varrho_{q}\left(a_{n+2 k} \cdot q^{k /(q-1)}\right) \cdot f \ddot{u} r$ jedes $k$ definiert. Es gilt

$$
\left\{\begin{aligned}
\varrho_{q}\left(a+\sum_{k=1}^{\infty} a_{n+2 k} \cdot q^{k /(q-1)}\right) & =\mathscr{P}^{-1}\left(\varrho_{q} a\right), & & \text { wenn q ungerade, } \\
& =S q^{-1}\left(\varrho_{q} a\right), & & \text { wenn } q=2 .
\end{aligned}\right.
$$

Dieser Satz ist spezieller als das Theorem 2 von Adams [2], da wir uns auf torsionsfreie $C W$-Komplexe $X$ eingeschränkt haben. ADAMs betrachtet nur $K^{0}(X)$, aber das genügt, um den Satz für $K^{*}(X)$ zu bekommen: Unte: Verwendung. der Bezeichnungen von $[4]$ hat man nämlich die kanonischen „Einhängungs"-Isomorphismen

$$
K^{1}(X)=\widetilde{K}^{0}\left(\boldsymbol{S}\left(X^{+}\right)\right), \quad H^{*}(X ; A)=\widetilde{H}^{*}\left(\mathbf{S}\left(X^{+}\right) ; A\right)
$$

( $A$ beliebiger Koeffizientenbereich).

Sowohl der Chernsche Charakter als' auch der Cohomologie-Automorphismus $\mathscr{P}^{-1}\left(\right.$ bzw. $\left.S q^{-1}\right)$ ist mit der ,Einhängung" vertauschbar. 
In der Formulierung des Satzes bei Adams kommt der kanonische AntiAutomorphismus der Steenrodschen Algebra vor. Bei Beachtung von 1.10 folgt unsere Formuliering . Es sei auch noch erwähnt, daB in einem torsionsfreien Raum $S q=\sum_{i=0}^{\infty} S q^{2 i}$ da $S q^{2 i+1}=S q^{1} S q^{2 i}$ und $S q^{1}$ der Bocksteinsche Operator ist, der in einem Raum ohne 2-Torsion verschwindet.

Ein Beispiel möge den Satz von Adams illustrieren. Fứr Elemente $x_{1}, \ldots, x_{s} \in H^{2}(X ; \boldsymbol{Z})$ gibt es genau ein $\xi \in K^{0}(X)$ mit

$$
\operatorname{ch}(\xi)=x_{1} \ldots x_{s} \cdot \prod_{j=1}^{s}\left(1-e^{-x_{j}}\right) / \underline{x}_{j} .
$$

Für dieses $\xi$ mit $a=x_{1} \ldots x_{s}$ folgt die Formel (4) leicht aus 2.9 (14) und aus

$$
\left.\mathscr{P}^{-1}\left(x_{j}\right)=x_{j}-x_{j}^{q}+x_{j}^{q^{2}}-\cdots \quad \text { (s. } 2.12\left(21^{\prime}\right)\right) .
$$

(Die Formel (7) bleibt bestehen, wenn $q=2$ ist und $\mathscr{P}$ durch $S q$ ersetzt wird.)

4.3. Wir möchten in diesem Abschnitt mit Hilfe des Satzes von ADAMS (4.2) zeigen, daß der Satz $3.2 \mathrm{im}$ Falle torsionsfreier Mannigfaltigkeiten für $\lambda=\mathscr{P}^{-1}$ aus dem ,differenzierbaren Riemann-Rochschen Satze" [3] folgt.

Die in diesem Abschnitt vorkommenden Mannigfaltigkeiten sollen kompakt, orientiert, differenzierbar sein und ihre ganzzahlige Cohomologie soll keine Torsion besitzen. $X$ sei eine solche Mannigfaltigkeit und $w_{2}(X) \in H^{2}\left(X ; \boldsymbol{Z}_{2}\right)$ ihre zweitc Stiefel-Whitneysche Klasse. Da $X$ torsionsfrei ist, kann jedes Element von $H^{2}\left(X ; \boldsymbol{Z}_{2}\right)$ durch Reduktion mod 2 aus einer ganzzahligen Klasse gewonnen werden. $c_{1}(X) \in H^{2}(X ; \boldsymbol{Z})$ sei für jede der in diesem Abschnitt zugelassenen Mannigfaltigkeiten $X$ festgewählt, und zwar so, daß die Reduktion mod 2 von $c_{1}(X)$ gleich $w_{2}(X)$ ist.

Die totale Toddsche Klasse $\mathscr{T}(X) \in H^{*}(X ; Q)$ wird dann mit Hilfe von $c_{1}(X)$ und der Pontrjaginschen Klassen $p_{i}(X)$ wie folgt definiert:

$$
\mathscr{T}(X)=e^{c_{1}(X) / 2} \cdot \sum_{j=0}^{\infty} \hat{A}_{j}\left(p_{1}(X), \ldots, p_{j}(X)\right) .
$$

Für eine stetige Abbildung $g: Y \rightarrow X$ zulässiger Mannigfaltigkeiten und rür $\eta \in K^{*}(Y)$ gilt:

$$
g_{*}(\operatorname{ch}(\eta) \cdot \mathscr{T}(Y))=\operatorname{ch}\left(g_{!} \eta\right) \cdot \mathscr{T}(X), \quad g_{!} \eta \in K^{*}(X) .
$$

Das ist der differenzierbare Riemann-Rochsche Satz [3,13]. Das Element $g, \eta$ von $K^{*}(X)$ ist durch (9) eindeutig bestimmt, da $X$ nach Voraussetzung keine Torsion hat $[4]$.

Es sei $q$ eine ungerade Primzahl und $f=q^{1 /(q-1)}$. Wir nehmen nun an, daß $c h(\eta)$ von der Gestalt (3) ist, also mit $a \in H^{n}(X ; \boldsymbol{Z})$ beginnt. Dann ist $\operatorname{ch}(g, \eta)=g_{*} a+$ höhere Glieder. Wir setzen

$$
m=\operatorname{dim} g_{*} a=n+\operatorname{dim} X-\operatorname{dim} Y .
$$


Es werde nun in (9) die $2 k$-dimensionale Komponente von $\mathscr{T}(Y)$ durch ihr Vielfaches mit $f^{k}$ ersetzt, ebenso für $\mathscr{T}(X)$. Die $(n+2 k)$-dimensionale Komponente von $\operatorname{ch}(\eta)$ werde durch ihr Vielfaches mit $f^{k}$ ersetzt, ebenso werde die $(m+2 k)$-dimensionale Komponente von $c h\left(g_{1} \eta\right)$ durch ihr Vielfaches mit $f^{k}$ ersetzt. Nachdem diese Ersetzungen geschehen sind, werde mod $q$ reduziert. Man erhält auf diese Weise

$$
g_{*}\left(\mathscr{P}^{-\mathrm{r}}\left(\varrho_{q} a\right) \mathrm{Wu}(\mathscr{P}, Y)\right)=\mathscr{P}^{-1}\left(g_{*}\left(\varrho_{q} a\right)\right)^{\Uparrow} \mathrm{Wu}(\mathscr{P}, X) .
$$

Man wendet hierzu (4) auf $\eta$ und $g_{!} \eta$ an, beachtet, daB $e^{f c_{1}(X) / 2} \equiv 1(\operatorname{mad} q)$, und benutzt 2.12 (20). Da $Y$ nach Voraussetzung keine Torsion hat, läBt sich jedes Element $y \in H^{n}\left(Y ; \boldsymbol{Z}_{q}\right)$ als $\varrho_{q} a$ mit $a \in H^{n}(Y ; \boldsymbol{Z})$ schreiben und jedes $a \in H^{n}(Y ; \boldsymbol{Z})$ tritt als Anfangsterm des Chernschen Charakters eines Elementes $\eta \in K^{*}(Y)$ auf [4]. Also liefert (10) den Satz 3.2 für alle $y \in H^{*}\left(Y ; \boldsymbol{Z}_{q}\right)$ im Falle des Cohomologie-Automorphismus $\lambda=\mathscr{P}^{-1}$

\section{§5. Über die Relationen zwischen den charakteristischen Zahlen}

5.1. Wir wollen zunächst die Relationen zwischen den Chernschen Zahlen untersuchen. Vgl. dazu den Bericht [12]. Eine schwache fastkomplexe Struktur auf einer kompakten differenzierbaren Mannigfaltigkeit $M$ ist eine schwache komplexe Struktur des Tangentialbündels von $M$, d.h. ein komplexes Vektorraum-Bündel über $M$, das als reelles Bündel isomorph zum Tangentialbündel von $M$ plus (Whitneysche Summe) einem trivialen reellen Vektorraum-Bündel ist $[5$, III, §2.1]. Eine schwach-fastkomplexe Mannigfaltigkeit ist kanonisch orientiert, hat also einen orientierten Grundzyklus [M]. Die Chernschen Klassen $c_{i}(M)$ sind per definitionem die des komplexen Vektorraum-Bündels, welches die schwache fastkomplexe Struktur bestimmt. Die Dimension von $M$ sei gerade $(\operatorname{dim} M=2 n)$. Für jede Partition $\pi=\left(i_{1}, \ldots, i_{r}\right)$ von $n$ bilde man das Monom $c_{i_{1}} \ldots c_{i_{r}} \in H^{2 n}(M ; Z)$. Der Wert dieses Monoms auf $[M]$ werde mit $c_{\pi}(M)$ bezeichnet. Die $c_{\pi}(M)$ sind ganze Zahlen und werden Chernsche Zahlen von $M$ genannt.

Es sei $B_{U}$ der klassifizierende Raum der unendlichen unitären Gruppe. $\left(B_{\boldsymbol{U}}=\lim _{n \rightarrow \infty} B_{\boldsymbol{U}(n)}\right)$. Dann ist

$$
H^{* * *}\left(B_{\boldsymbol{U}} ; \boldsymbol{Z}\right)=\boldsymbol{Z}\left[\left[c_{1}, c_{2}, \ldots\right]\right],
$$

wo $c_{i}$ die , universelle“ $2 i$-dimensionale Chernsche Klasse ist. Man hat einen Homomorphismus

$$
\alpha(M): H^{2 n}\left(B_{\boldsymbol{U}} ; \boldsymbol{Z}\right) \rightarrow \boldsymbol{Z}
$$

wobei $\alpha(M)\left(c_{i_{1}} \ldots c_{i_{r}}\right)=c_{\pi}(M),\left(\pi=\left(i_{1}, \ldots, i_{r}\right), i_{1}+\cdots+i_{r}=n\right)$.

Es sei $q$ eine Primzahl. Nach Reduktion $\bmod q$ kann die Chernsche Klasse $c_{i}$ als Element von $H^{2 i}\left(B_{\boldsymbol{U}} ; \boldsymbol{Z}_{q}\right)$ aufgefaßt werden. Es ist dann

$$
H^{* *}\left(B_{\boldsymbol{U}} ; \boldsymbol{Z}_{q}\right)=\boldsymbol{Z}_{q}\left[\left[c_{1}, c_{2} ; \ldots\right]\right],
$$


und wir haben vermöge (2) einen Homomorphismus

$$
\alpha(M)_{q}: H^{2}{ }^{2}\left(B_{\boldsymbol{C}} ; \boldsymbol{Z}_{q}\right) \rightarrow \boldsymbol{Z}_{q} .
$$

Man nennt ein Element a von $H^{2 n}\left(B_{\boldsymbol{U}} ; \boldsymbol{Z}_{q}\right)$ eine ,Relation zwischen Chernschen Zahlen $\bmod q^{\prime s}$, wenn $a$ für alle kompakten differenzierbaren schwach-fastkomplexen Mannigfaltigkeiten $M$ der reellen Dimension $2 n$ zum Kern von $\alpha(M)_{q}$ gehört. Diese Relationen bilden eine Untergruppe von $H^{2 n}\left(B_{\boldsymbol{V}} ; \boldsymbol{Z}_{q}\right)$, die wir mit $R^{2 n}\left(B_{\boldsymbol{U}} ; \boldsymbol{Z}_{q}\right)$ bezeichnen. $R^{* *}\left(B_{\boldsymbol{U}} ; \boldsymbol{Z}_{q}\right)$ sei die Untergruppe derjenigen Elemente von $H^{* *}\left(B_{\boldsymbol{U}} ; \boldsymbol{Z}_{q}\right)$, deren $2 n$-dimensionale Komponente für jedes $n$ zu $R^{2 n}\left(B_{U} ; \boldsymbol{Z}_{q}\right)$ gehört.

5.2. In $H^{* *}\left(B_{\boldsymbol{V}} ; \boldsymbol{Z}_{q}\right)$ liegt die universelle totale Chernsche Klasse $c=$ $1+c_{1}+c_{2}+\cdots$. Mit $\left(c^{-1}\right)_{i}$ werde die $2 i$-dimensionale Komponente vori $c^{-1}$ bezeichnet. Durch die Festsetzungen

$$
c_{i} \rightarrow\left(c^{-1}\right)_{i}
$$

erhält man einen multiplikativen gradtreuen involutorischen Automorphismus $D$ von $H^{* *}\left(B_{\boldsymbol{V}} ; \boldsymbol{Z}_{q}\right)$, vgl. [8]. Für eine multiplikative Folge

$$
\sum_{j=0}^{\infty} K_{j}\left(c_{\mathbf{1}}, \ldots, c_{j}\right) \in H^{* *}\left(B_{\boldsymbol{U}} ; \boldsymbol{Z}_{q}\right)
$$

ist

$$
D\left(\sum_{j=0}^{\infty} K_{j}\left(c_{1}, \ldots, c_{j}\right)\right)=\sum_{j=0}^{\infty} K_{j}\left(D c_{1}, \ldots, D c_{j}\right)=\left(\sum_{j=0}^{\infty} K_{j}\left(c_{1}, \ldots, c_{j}\right)\right)^{-1}
$$

$D$ ist mit jedem Cohomologie-Automorphismus $\lambda$ vertauschbar, da $\lambda(c) \in$ $H^{* *}\left(B_{\boldsymbol{U}} ; \boldsymbol{Z}_{q}\right)$ eine multiplikative Folge ist (mit der charakteristischen Potenzreihe $1+\lambda x)$.

Der Automorphismus $D$ entspricht dem Übergang von einem Bündel $\xi$ zu dem inversen Bündel $\eta$ von $\xi$. $([\xi]+[\eta]=0$, s. $[5$, III, §1.3].) Der Automorphismus $D$ wird also durch eine "Abbildung" $B_{\boldsymbol{U}} \rightarrow B_{\boldsymbol{U}}$ induziert. Man kann $D$ auffassen als den kanonischen Anti-Automorphismus der Hopfschen Algebra $H^{* * *}\left(B_{\boldsymbol{U}} ; \mathbb{Z}_{q}\right)$, vgl. [1].

5.3. Für einen Cohomologie-Automorphismus $\lambda$ ist $\mathrm{Wu}(\lambda)$ ein multiplikativer Funktor, zu dem eine multiplikative Folge $\left\{K_{j}\left(c_{1}, \ldots, c_{j}\right)\right\}$ gehört (s. 2.6). Das Element $\sum_{j=0}^{\infty} K_{j}\left(c_{\boldsymbol{1}}, \ldots, c_{j}\right) \in H^{* *}\left(B_{\boldsymbol{U}} ; \boldsymbol{Z}_{q}\right)$ soll ebenfalls mit Wu $(\lambda)$ bezeichnet werden. Dann gilt

$$
\lambda y-y \cdot \mathrm{Wu}(\lambda) \in R^{* *}\left(B_{\boldsymbol{U}} ; \boldsymbol{Z}_{q}\right) \quad \text { für alle } y \in H^{* *}\left(B_{\boldsymbol{U}} ; \mathbb{Z}_{q}\right) \text {. }
$$

Die Aussage (7) ist eine Konsequenz der Formel 3.5 (17), wenn man diese auf eine beliebige schwach-fastkomplexe Mannigfaltigkeit $M$ anwendet, wobei das $y$ von 3.5 (17) alle Polynome in den Chernschen Klassen von $M$ durchlauft.

Ordnet man jedem $y \in H^{* *}\left(B_{\boldsymbol{U}} ; \boldsymbol{Z}_{q}\right)$ die $2 n$-dimensionale Komponente von (7) zu, dann erhält man einen additiven Homomorphismus von $H^{* *}\left(B_{\mathbf{U}} ; \mathbb{Z}_{g}\right)$ 
in $R^{2 n}\left(B_{\boldsymbol{U}} ; \boldsymbol{Z}_{q}\right)$, dessen Bild mit $R^{2 n}\left(B_{\boldsymbol{U}} ; \boldsymbol{Z}_{q} ; \lambda\right)$, bezeichnet werde. Die $m$-dimensionale Komponente einer Cohomologie-Klasse $a$ werde gelegentlich durch $a_{m}$ angedeutet, (obwohl das mit den Bezeichnungen der Chernschen und Pontrjaginschen Klassen nicht übereinstimmt). Die Untergruppe $\boldsymbol{R}^{2 n}\left(B_{\boldsymbol{U}} ; \boldsymbol{Z}_{q} ; \lambda\right)$ von $R^{2 n_{n}}\left(B_{\boldsymbol{U}} ; \boldsymbol{Z}_{q}\right)$ läßt sich auf verschiedene Weisen beschreiben:

Lemia. $R^{2 n}\left(B_{\boldsymbol{U}} ; \boldsymbol{Z}_{q} ; \lambda\right)=$

$$
\begin{aligned}
& \left\{\left(\lambda y \cdot \mathrm{Wu}\left(\lambda^{-1}\right)-y\right)_{\mathbf{2}_{n}} \mid y \in H^{* *}\left(B_{\boldsymbol{U}} ; \boldsymbol{Z}_{q}\right)\right\}= \\
& \left\{\left(\lambda^{-1} y \cdot \mathrm{Wu}(\lambda)-y\right)_{2 n} \mid y \in H^{* *}\left(B_{\boldsymbol{U}} ; \boldsymbol{Z}_{q}\right)\right\}=R^{2 n}\left(B_{\boldsymbol{U}} ; \boldsymbol{Z}_{q} ; \lambda^{-1}\right)= \\
& \left\{D(\lambda y \cdot \underline{\lambda}-y)_{2 n} \mid y \in H^{* *}\left(B_{\boldsymbol{U}} ; \boldsymbol{Z}_{q}\right)\right\} .
\end{aligned}
$$

BewEIs. $\mathrm{Zu}$ (i) gelangt man, indem man in (7) y durch $y \cdot \mathrm{Wu}(\lambda)^{-1}$ ersetzt und beachtet, daß $\lambda\left(\mathrm{Wu}(\lambda)^{-1}\right)=\mathrm{Wu}\left(\lambda^{-1}\right)$, s. 2.8. Man bekommt (ii) aus (7) dadurch, daß man $y$ durch $\lambda^{-1} y$ ersetzt. Im Falle (iii) werde zunächst an den Funktor $\underline{\lambda}$ erinnert, der eine multiplikative Folge von Polynomen bestimmt und damit ein Element von $H^{* *}\left(B_{\boldsymbol{U}} ; \boldsymbol{Z}_{q}\right)$, s. 2.6. Nun folgt (iii) aus (i) wegen der Vertauschbarkeit von $D$ mit $\lambda$ und da $D \underline{\lambda}=(\underline{\lambda})^{-1}=\mathrm{Wu}\left(\lambda^{-1}\right)$.

5.4. Mrlnor führt in [17] das stabile Objekt $M \boldsymbol{U}$ ein (stabiler universeller Thomscher Raum für die unitầre Gruppe). Man hat den Thomschen Isomorphismus

$$
\Phi: H^{* *}\left(B_{\boldsymbol{U}} ; \boldsymbol{Z}_{q}\right) \rightarrow H^{* *}\left(M \boldsymbol{U} ; \boldsymbol{Z}_{q}\right),
$$

welcher per definitionem im hier betrachteten stabilen Fall die Graduierung erhält. $\mathrm{Zu}$ einem Cohomologie-Automorphismus $\lambda$ gehört der multiplikative Funktor $\lambda$, welcher in üblicher Weise als Element von $H^{* *}\left(B_{\mathbf{U}} ; \boldsymbol{Z}_{q}\right)$ aufzufassen ist (2.6). Die Steenrodsche Algebra $S^{* *}$ (für die Cohomologie mit Koeffizienten in $\left.\boldsymbol{Z}_{q}\right)$ operiert auf $H^{* *}\left(M \boldsymbol{U} ; \boldsymbol{Z}_{q}\right)$. Für den Cohomologie-Automorphismus $\lambda \in S^{* *}$ gilt:

$$
\lambda(\Phi y)=\Phi(\lambda y \cdot \underline{\lambda}) \quad \text { für } y \in H^{* *}\left(B_{U} ; Z_{q}\right) .
$$

Die Formel (9) ist das stabile Gegenstück zu 2.15 (24). Aus 5.3 (iii) folgt sofort

$$
\left\{\begin{aligned}
\Phi D R^{2 n}\left(B_{\boldsymbol{U}} ; \boldsymbol{Z}_{q} ; \lambda\right) & =\left\{(\lambda y-y)_{2 n} \mid y \in H^{* *}\left(M \boldsymbol{U} ; \boldsymbol{Z}_{q}\right)\right\} \\
& =\left\{(\lambda y)_{2 n} \mid y \in \sum_{i=0}^{n-1} H^{2 i}\left(M \boldsymbol{U} ; \boldsymbol{Z}_{q}\right)\right\} .
\end{aligned}\right.
$$

Nun hat MiLnor in [17] gezeigt, daß die Algebra $S^{*} /(\delta)$ frei auf $H^{*}\left(M \boldsymbol{U} ; \boldsymbol{Z}_{q}\right)$ operiert. Hierbei ist $S^{*}$ die Steenrodsche Algebra und $(\delta)$ das von dem Bocksteinschen Operator $\delta$ erzeugte Ideal $\left(\delta=S q^{\mathbf{1}}\right.$ für $\left.q=2\right)$. Man wähle eine Basis vòn $H^{*}\left(M \boldsymbol{U} ; \boldsymbol{Z}_{q}\right)$ über $S^{*} /(\delta)$ aus homogen-dimensionalen Elementen. Die Anzahl der Basis-Elemente der Dimension $2 n$ ist unabhängig von der Wahl der Basis und werde mit $b(n ; q)$ bezeichnet. Wählt man in (10) für $\lambda$ den Steenrodschen Cohomologie-Automorphismus $\mathscr{P}$ (bzw. Sq), dann folgt 
unmittelbar

$$
\left\{\begin{array}{c}
\operatorname{dim}_{\boldsymbol{Z}_{q}} R^{2 n}\left(B_{\boldsymbol{U}} ; \boldsymbol{Z}_{q} ; \lambda\right)=\operatorname{dim}_{\boldsymbol{Z}_{q}} H^{2 n}\left(B_{\boldsymbol{V}} ; \boldsymbol{Z}_{q}\right)-b(n ; q) \\
\text { für } \lambda=\mathscr{P} \quad \text { bzw. } \lambda=S q .
\end{array}\right.
$$

5.5. Schreibt man die ganzzahligen Chernschen Klassen $c_{i}$ formal als elementar-symmetrische Funktionen in Variablen $x_{1}, x_{2}, \ldots, x_{N}$ ( $N$ groB) vom Grade 2, dann kann man für jede Partition $\pi=\left(i_{1}, \ldots, i_{r}\right)$ von $n$ das Element

$$
s(\pi)=\sum x_{1}^{i_{1}} \ldots x_{\tau}^{i_{\tau}} \in H^{2 n}\left(B_{\boldsymbol{U}} ; \boldsymbol{Z}\right)
$$

einführen. $s(\pi)$ ist die Summe aller paarweise verschiedenen Monome, die aus $x_{1}^{i_{1}} \ldots x_{r}^{i_{r}}$ durch Permutationen der $N$ Variablen entstehen (s. z.B. [11, §1.4]). Für eine schwach-fastkomplexe Mannigfaltigkeit $M$ der reellen Dimension $2 n$ definieren wir die ganze $Z$ ahl $s_{\pi}(M)$ als Wert von $s(\pi)$ auf dem orientierten Grundzyklus von $M$. Für die Partition $\pi=(n)$ setzen wir $s(M)=s_{\pi}(M)$. Wenn $n=2 k$, dann stimmt $s(M)$ mit der in 3.6 definierten ganzen $Z a h l s(M)$ überein. Genau wie in 3.6 können wir für eine schwachfastkomplexe $M$ der reellen Dimension $2 n$ nachweisen, daß $s(M)$ durch $q$ teilbar ist, wenn $n+1$ eine Potenz der Primzahl $q$ ist.

MrLNor hat in [17] eine Folge $M_{1}, M_{2}, M_{3}, \ldots$ von projektiv-algebraischen Mannigfaltigkeiten der komplexen Dimension $1,2,3, \ldots$ usw. angegeben, für die gilt:

$$
\begin{aligned}
& \text { i) } s\left(M_{n}\right)=1 \text {, wenn } n+1 \text { keine Primzahlpotenz ist, } \\
& \text { ii) } s\left(M_{n}\right)=q \text {, wenn } n+1 \text {, eine Potenz der Primzahl } q \text { ist. }
\end{aligned}
$$

Aus dèr Existénz dieser Mannigfaltigkeiten schließt man, daß

$$
\operatorname{dim}_{\boldsymbol{Z}_{q}} H^{2 n}\left(B_{\boldsymbol{U}} ; \boldsymbol{Z}_{q}\right) / \operatorname{dim}_{\boldsymbol{Z}_{q}} R^{2 n}\left(B_{\mathbf{U}} ; \boldsymbol{Z}_{q}\right) \geqq a(n ; q),
$$

wo $a(n ; q)$ die Anzahl der Partitionen von $n$ ist, die keinen Summanden der Form $q^{i}-1$ enthalten. Man benutzt dazu die Thomsche Formel

$$
s_{\omega}(M)=\sum_{\left\{\omega_{1}, \omega_{2}\right\}} s_{\omega_{1}}\left(M^{\prime}\right) \cdot s_{\omega_{2}}\left(M^{\prime \prime}\right)
$$

wo $M, M^{\prime}, M^{\prime \prime}$ schwach-fastkomplexe Mannigfaltigkeiten der reellen Dimensionen $2 n, 2 r, 2 s$ sind, $M=M^{\prime} \times M^{\prime \prime}, n=r+s$, und wo $\omega$ eine Partition von $n$ ist und $\left(\omega_{1}, \omega_{2}\right)$ alle möglichen Zerlegungen von $\omega$ in eine Partition $\omega_{1}$ von $r$ und eine Partition $\omega_{2}$ von $s$ durchläuft. (Die Formel (13) wird in [22, Chap. IV, $\$ 7]$ in einem etwas anderen Fall bewiesen.)

Nach MILnor $[17]$ ist $a(n ; q)=b(n ; q)$. Aus (11) und (12) folgt für $\lambda=\mathscr{P}$ bzw. $\lambda=S q$, daB $R^{2 n}\left(B_{\boldsymbol{U}} ; \boldsymbol{Z}_{q} ; \lambda\right)=R^{2 n}\left(B_{\boldsymbol{U}} ; \boldsymbol{Z}_{q}\right)$. Wir fassen das Resultat in folgendem Satz zusammen.

5.6. Satz. Für eine gegebene Primzahl $q$ sei $R^{2 n}\left(B_{\boldsymbol{U}} ; \boldsymbol{Z}_{q}\right)$ die Gruppe der Relationen mod $q$, die zwischen den Chernschen Zahlen schiach-fasikomplexer Mannigfaltigkeiten der reellen Dimension $2 n$ bestehen. $R^{2 n}\left(B_{U} ; Z_{q} ; \lambda\right)$ sei die Untergruppe derjenigen Relationen, die sich mit Hilfe der Wuschen Methode (7) 
und des Cohomologie-Automorplismus $\lambda$ (für die Cahomologie mit Koeffizienten $\boldsymbol{Z}_{q}$ ) erhalten lassen. Im allgemeinen ist $R^{2 n}\left(B_{\boldsymbol{U}} ; \boldsymbol{Z}_{q} ; \lambda\right)$ eine echte Untergruppe von $R^{2 n}\left(B_{\boldsymbol{v}} ; \boldsymbol{Z}_{q}\right)$. Wählt man jedoch den Steenrodschen Cohomologie-Automorphismus $\mathscr{P}$ ( $q$ ungerade) bzw. $S q$ (für $q=2$ ), dann gilt

$$
R^{2 n}\left(B_{\boldsymbol{U}} ; \boldsymbol{Z}_{q} ; \mathscr{P}\right)=R^{2 n}\left(B_{\boldsymbol{U}} ; \boldsymbol{Z}_{q}\right) \quad \text { bzw. } \quad R^{2 n}\left(B_{\boldsymbol{U}} ; \boldsymbol{Z}_{2} ; S q\right)=R^{2 n}\left(B_{\boldsymbol{U}} ; \boldsymbol{Z}_{2}\right) .
$$

Die Wuschen Relationen sind also vollständig. Die Dimension von $R^{2 n}\left(B_{\boldsymbol{V}} ; \mathbf{Z}_{q}\right)$ über $\boldsymbol{Z}_{q}$ ist gleich der Anzahl der Partitionen von n, die wenigstens einen Summanden der Form $q^{i}-1$ enthalten.

5.7. In 5.1 (2) haben wir den Homomorphismus $\alpha(M): H^{2 n}\left(B_{\boldsymbol{U}} ; \boldsymbol{Z}\right) \rightarrow \boldsymbol{Z}$ eingeführt. Hom $\left(H^{2 n}\left(B_{\boldsymbol{V}} ; \boldsymbol{Z}\right), \boldsymbol{Z}\right)=H_{2 n}\left(B_{\boldsymbol{U}} ; \boldsymbol{Z}\right)$. ist eine freie abelsche Gruppe, deren Rang gleich der Anzahl der Partitionen von $n$ ist. Die Menge der $\alpha(M)$, wo $M$ alle schwach-fastkomplexen Mannigfaltigkeiten der reellen Dimension $2 n$ durchläuft, bildet eine Untergruppe von $H_{2 n}\left(B_{\boldsymbol{U}} ; \boldsymbol{Z}\right)$ von endlichem Index. Diese Untergruppe kann durch Kongruenzen zwischeñ den Chernschen Zahlen angegeben werden. Diese Kongruenzen sind im Prinzip berechenbar (MrLnor [17, Part II]). Für eine vollständige Liste der Kongruenzen für $n \leqq 4$ s. [12]. Der Homomorphismus $\alpha(M): H^{2 n}\left(B_{\mathbf{v}} ; \boldsymbol{Z}\right) \rightarrow \boldsymbol{Z}$ induziert einen Homomi ¿phismus

$$
\alpha(M)_{\boldsymbol{Q}}: H^{2 n}\left(B_{\boldsymbol{U}} ; \boldsymbol{Q}\right) \rightarrow \boldsymbol{Q} .
$$

Es sei $R^{2 n}\left(B_{\boldsymbol{V}} ; \boldsymbol{Q} / \boldsymbol{Z}\right)$ die Untergruppe derjenigen Elemente von $H^{2 n}\left(B_{\boldsymbol{V}} ; \boldsymbol{Q}\right)$, auff denen $\alpha(M)_{\boldsymbol{Q}}$ für jede schwach-fastkomplexe $M$ der reellen Dimension $2 n$ ganzzahlige Werte annimmt. $R^{2 n}\left(B_{\boldsymbol{V}} ; \boldsymbol{Q} / \mathbf{Z}\right)$ ist die Gruppe der Kongruenzen zwischen den Chernschen Zahlen schwach-fastkomplexer Mannigfaltigkeiten der reellen Dimension $2 n$. Es sei $R^{* *}\left(B_{\mathbf{V}} ; \boldsymbol{Q} / \mathbf{Z}\right)$ die Gruppe der Elemente von $H^{* *}\left(B_{\boldsymbol{V}} ; \boldsymbol{Q}\right)$, deren $2 n$-dimensionale Komponenten für jedes $n \mathrm{zu}$ $R^{2 n}\left(B_{\boldsymbol{U}} ; \boldsymbol{Q} / \mathbf{Z}\right)$ gehören.

Schreibt man die ganzzahligen Chernschen Klassen wieder formal als elementar-symmetrische 'Funktionen in den Variablén $x_{1}, \ldots, x_{N}(N$ groß) vom Grade 2, dann kann man die elementar-symmetrischen Funktionen $e_{1}, e_{2}, \ldots$ in den $1-e^{-x_{i}}$ einführen. Das sind wohldefinierte Elemente von $H^{* *}\left(B_{\boldsymbol{U}} ; \boldsymbol{Q}\right)$. Den von $e_{0}=1, e_{1}, e_{2}, \ldots$ ïber $\boldsymbol{Z}$ erzeugten Unterring von $H^{* *}\left(B_{\boldsymbol{U}} ; \boldsymbol{Q}\right)$ bezeichnen wir mit $\operatorname{ch}\left(B_{\boldsymbol{V}}\right)$. Es sei $\mathscr{T}$ die universelle Toddsche Klasse

$$
\mathscr{T}=\sum_{j=0}^{\infty} T_{i}\left(c_{1}, \ldots, c_{j}\right) \in H^{* *}\left(B_{\boldsymbol{U}} ; \boldsymbol{Q}\right) .
$$

Satz. Für jedes $z \in \operatorname{ch}\left(B_{\boldsymbol{U}}\right)$ gehört $z \cdot \mathscr{T}-z u \cdot R^{* *}\left(B_{\boldsymbol{U}} ; \boldsymbol{Q} / \mathbf{Z}\right)$.

Der Satz ist eine unmittelbare Folgerung aus bekannten Ganzzahligkeitssätzen im Zusammenhang mit dem Satz von Riemann-Roch (s. [5, Part III, Theorem 3.6] oder [3]). Man hat zu beachten, daß $z \in \operatorname{ch}\left(B_{\boldsymbol{U}}\right)$ für jede schwachfastkomplexe $M$ ein Element von $\operatorname{ch} K(M)$ liefert, (das zum komplexen Tangentialbündel ver̂möge einer Darstellung der unitären Gruppe assoziiert ist). 
VeRMUTUNG. $R^{2 n}\left(B_{\boldsymbol{U}} ; \boldsymbol{Q} / \boldsymbol{Z}\right)=\left\{(z \cdot \mathscr{T})_{2 n} \mid z \in \operatorname{ch}\left(B_{\boldsymbol{U}}\right)\right\}$, wo $(z \cdot \mathscr{T})_{2 n}$ die $2 n$ dimensionale Komponente von $z \cdot \mathscr{T}$ bezeichnet.

Wir können diese Vermutung nicht beweisen. Jedoch werden wir zeigen, daß die $(z \cdot \mathscr{T})_{2 n}$ für jede Primzahl $q$ alle Relationen mod $q$ liefern. Die ungelöste Frage ist dann die nach den Relationen modulo den Potenzen einer Prim$z a h l$. Wir betrachten ein Monom $e_{i_{1}} e_{i_{2}} \ldots e_{i_{r}}$, wo $\left(i_{1}, i_{2}, \ldots, i_{n}\right)$ eine Partition einer Zahl $k<n$ ist. Für $k=0$ haben wir nur das Monom 1. Das Monom $e_{i_{1}} \ldots e_{i_{r}}$ ist ein Element von $H^{* *}\left(B_{\boldsymbol{U}} ; Q\right)$, das mit $c_{i_{1}} \ldots c_{i_{r}}$ beginnt. Ersetzt man in $e_{i_{1}} \ldots e_{i_{r}}$ die Komponente der Dimension $2 k+2 j$ durch ihr Vielfaches mit $f^{j}$, wo $f=q^{1 /(q-1)}$ und reduziert modulo $q$, dann geht $e_{i_{1}} \ldots e_{i_{r}}$ in $\mathscr{P}^{-1}\left(c_{i_{1}} \ldots c_{i_{r}}\right)$ iiber. Das ist eine rein formale Tatsache im Zusammenhang mit dem Satz von Adams (s. $4.2(6)$ ). (Für $q=2$ ist $\mathscr{P}$ durch $S q$ zu ersetzen.) Ersetzt man ferner in $\mathscr{T}$ die $2 j$-dimensionale Komponente durch ihr Vielfaches mit $f \dot{ }$, darin geht $\mathscr{T}$ in $W u(\mathscr{P})$ bzw. Wu $(S q)$ durch Reduktion mod $q$ über. (Siehe 2.9, $2.11,2.13$.) Aus 5.3 (ii) und 5.6 folgt unmittelbar

SATz. Die Riemann-Roch-Kongruenzen $z \cdot \mathscr{T} \in R^{* *}\left(B_{\boldsymbol{U}} ; Q / Z\right), z \in \operatorname{ch}\left(B_{\boldsymbol{U}}\right)$, implizieren alle Relationen mod $q$ zwischen den Chernschen Zahlen.

5.8. Für die Pontrjaginschen Zahlen einer orientierten kompakten differenzierbaren Mannigfaltıgkeit $M$ gelten ähnliche Resultate, wie wir sie für die Chernschen Zahlen besprochen haben. Es sei $B_{o}$ der klassifizierende Raum für die unendliche orthogonale Gruppe. Ist $q$ eine ungerade Primzahl, dann gilt

$$
H^{* *}\left(B_{\boldsymbol{o}} ; \boldsymbol{Z}_{q}\right)=\boldsymbol{Z}_{q}\left[\left[p_{1}, p_{2}, \ldots\right]\right],
$$

wo $p_{i} \in H^{4 i}\left(B_{\boldsymbol{O}} ; \boldsymbol{Z}_{q}\right)$ die Reduktion $\bmod q$ der universellen ganzzahligen Pontrjaginschen Klasse der Dimension $4 i$ ist. Für jede orientierte kompakte differenzierbare $M$ sind die Pontrjaginschen Zahlen definiert. Das sind ganze Zahlen. Reduktion mod $q$ der Pontrjaginschen Zahlen liefert einen Homomorphismus

$$
\alpha(M)_{q}: H^{4 k}\left(B_{\boldsymbol{Q}} ; \boldsymbol{Z}_{q}\right) \rightarrow \boldsymbol{Z}_{q}, \quad \operatorname{dim} M=4 k .
$$

$R^{4 k}\left(B_{\boldsymbol{O}} ; \boldsymbol{Z}_{q}\right)$ sei die Untergruppe der Elemente von $H^{4 k}\left(B_{\boldsymbol{O}} ; \boldsymbol{Z}_{q}\right)$, welche für jede $4 k$-dimensionale orientierte kompakte differenzierbare $M$ zum Kern von $\alpha(M)_{q}$ gehören. $R^{4 k}\left(B_{\boldsymbol{O}} ; \boldsymbol{Z}_{q}\right)$ ist die Gruppe der Relationen mod $q$ zwischen den Pontrjaginschen Zahlen in der Dimension $4 k$.

SATz. Es sei $\mathscr{P}$ der Steenrodsche Cohomologie-Automorphismus für die Cohomologie mit Koeffizienten in $\boldsymbol{Z}_{q}$ (qungerade Primzahl). Der multiplikative Funktor $\mathrm{Wu}(\mathscr{P})$ ist ein Element von $H^{* *}\left(B_{\boldsymbol{O}} ; \boldsymbol{Z}_{q}\right)$. Es gilt

$$
R^{4 k}\left(B_{\boldsymbol{O}} ; \boldsymbol{Z}_{q}\right)=\left\{(\mathscr{P} y-y \cdot \mathrm{Wu}(\mathscr{P}))_{4 k} \mid y \in H^{* *}\left(\mathcal{B}_{\boldsymbol{O}} ; \boldsymbol{Z}_{q}\right)\right\}
$$

Die Relationen, die sich mit Hilfe der Wuschen Methode gevinnen lassen (3.5 (17)), liefern also ganz $R^{4 k}\left(B_{\boldsymbol{O}} ; \boldsymbol{Z}_{q}\right)$. Die Dimension von $R^{4 k}\left(B_{\boldsymbol{o}} ; \boldsymbol{Z}_{q}\right)$ ist gleich der. Anzahl der Partitionen von $2 k$, die nur gerade Summanden enthalten und fiur die wenigstens ein Summand von der Form $q^{i}-1$ ist. 
Der Beweis erfolgt analog wie im Fall der Chernschen Zahlen unter wesentlicher Benutzung der Arbeit [17] von Milnor. Man kann wie in 5.7 die Gruppe $R^{\mathbf{4} k}\left(B_{\boldsymbol{O}} ; \boldsymbol{Q} / \boldsymbol{R}\right)$ aller Kongruenzen zwischen den Pontrjaginschen Zahlen einführen, eine analoge Vermutung formulieren und aurh eine dem letzten Satz von 5.7 entsprechende Behauptung beweisen.

5.9. Schließlich gelten ähnliche Überlegungen für die Stiefel-Whitneyschen Zahlen von nicht notwendigerweise orientierbaren Mannigfaltigkeiten: Diese Zahlen sind Elemente von $\boldsymbol{Z}_{2}$. Auch hier gilt die Vollständigkeit der Wuschen Relationen, was von Dold bewiesen wurde [8], dessen Beweismethode wir im $\$ 5$ der vorliegenden Arbeit ganz entscheidend benutzt haben.

\section{Literatur}

[1] Adams, J. F.: On formulae of Thom and Wu. (erscheint demnächst).

[2] - On Chern characters and the structure of the unitary group. Proc. Cambr. Phil. Soc, 57, 189-199 (1961).

[3] AтIYAh, M. F., and F. Hirzebruch: Riemann-Roch theorems for differentiable manifolds. Bull. Amer. Math. Soc. 65, 276-281 (1959).

[4] - , and F. HiRzEBRUCH: Vector bundles and homogeneous spaces. Differential Geometry. Proceedings of Symposia in Pure Mathematics, vol. 3, American Mathematical Society 1961.

[5] Borel, A., and F. Hirzebruch: Characteristic classes and homogeneous spaces I, II, III. Amer J. Math. 80, 458- 538 (1958); 81, 315-382 (1959); 82, 491-504 (1960).

[6] -, et J.P. Serre: Le théorème de Riemann-Roch (d'après Grothendieck). Bull. Soc. Math. France 86, 97-136 (1958).

[7] Cartan, 'H.: Sur les groupes d'Eilenberg-MacLane, I et II. Proc. Nat. Acad. Sci. U.S.A. 40, 467-471 und 704-707 (1954).

[8] Dold, A.: Vollständigkeit der Wuschen Relationen zwischen den Stiefel-Whitneyschen Zahlen differenzierbarer Mannigfaltigkeiten. Math. Z. 65, 200-206 (1956).

[9] Eilenderg, S., and N. Steenrod: Foundations of algebraic topology. Princeton Mathematical Series 15, Princeton University Press 1952.

[10] Hirzebruch; F.: On Steenrod's reduced powers, the index of inertia, and the Todd genus. Proc. Nat. Acad. Sci. U.S.A. 39, $951-956$ (1953).

[11] - Neue topologišche Methoden in dex algebraischen Geometrie. Ergebnisse der Mathematik. Neue Folge, Heft 9. Berlin-Göttingen-Heidelberg 1956.

[12] - Komplexe Mannigfaltigkeiten. Proc. of the Intern. Congr. of Math. 1958, Cambridge University Press 1960, pp. 119-136.

[13] - A Riemann-Roch theorem for differentiable manifolds. Séminaire Bourbaki, Exp. 177, Février 1959 .

[14] Milnor, J.: The geometric realization of a semi-simplicial complex. Ann. Math. (2) $65,357-362(1957)$.

[15] - Lectures on characteristic classes. Mimeographed notes. Princeton 1957.

[16] - The Steenrod algebra and its dual. Ann. Math. 67, 150-171 (1958).

[17] - On the cobordism ring $\Omega^{*}$ and a complex analogue, Part I. Amer. J. Math. 82, 505-521 (1960). Part II erscheint demnächst.

[18]. Puppe, D.: Homotopiemengen und ihre induzierten Abbildungen I. Math Z. 69, $299-344$ (1958).

[19] Serre, J. P.: Cohomologie modulo 2 des complexes d'Eilenberg-MacLane. Comm. Math. Helv. 27, 198-232 (1953).

[20] Steenrod, N.: Homology groups of symmetric groups and reduced power operations. Proc. Nat. Acad. Sci. U.S.A. 39, 213-223 (1953). 
[21] - Cohomology operations and obstructions to extending continuous functions. Colloquium lectures. Mimeographed notes. Princeton 1957 .

[22] Tном, R. : Quelques propriétés globales des variétés différentiables Comm. Math. Helv. 28, 17-86 (1954).

[23] Uspensky, J. V., and M. A. Heaslet: Elementary Number Theory. New York 1939.

[24] Whitehead, J.H.C.: Combinatorial homotopy I. Bull. Amer. Math. Soc. 55, $213-245(1949)$.

[25] WU WEN-TSUN: Classes caractéristiques et i-carrés d'une variété. C. R. Acad. Sci., Paris 230, $508-511$ (1950).

[26] - Sur les puissances de Steenrod. Colloque de Topologie de Strasbourg 1951 (vervielfältigt).

Mathematisches Institut der Universität, Bonn

Pembroke College, Cambridge

Mathematical Instituie, Oxford

(Eingegangen am 10. April 1961) 